\title{
Scalar field in the Bianchi I: Non commutative classical and Quantum Cosmology
}

\author{
J. Socorro ${ }^{1,2} *$ Luis O. Pimentel ${ }^{3} \dagger$ C. Ortiz ${ }^{1,4}+$ and M. Aguero $\$$ \\ ${ }^{1}$ Departamento de Física, DCI-Campus León, Universidad de Guanajuato, \\ A.P. E-143, C.P. 37150, León, Guanajuato, México \\ ${ }^{2}$ Facultad de Ciencias de la Universidad Autónoma del Estado de México, \\ Instituto Literario No. 100, Toluca, C.P. 50000, Edo de Mex, México \\ ${ }^{3}$ Departamento de Física, Universidad Autónoma Metropolitana, Apartado Postal 55-534, 09340, México, D.F. \\ ${ }^{4}$ Unidad Académica de Física, Universidad Autónoma de Zacatecas \\ Cda. Solidaridad SN, CP. 98060, Zacatecas, Zac., México
}

(Dated: September 23, 2021)

\begin{abstract}
Using the ADM formalism in the minisuperspace, we obtain the commutative and noncommutative exact classical solutions and exact wave function to the Wheeler-DeWitt equation with an arbitrary factor ordering, for the anisotropic Bianchi type I cosmological model, coupled to a scalar field, cosmological term and barotropic perfect fluid. We introduce noncommutative scale factors, considering that all minisuperspace variables $q^{i}$ do not commute, so the symplectic structure was modified. In the classical regime, it is shown that the anisotropic parameter $\beta_{ \pm \mathrm{nc}}$ and the field $\phi$, for some value in the $\lambda_{\text {eff }}$ cosmological term and noncommutative $\theta$ parameter, present a dynamical isotropization up to a critical cosmic time $t_{c}$; after this time, the effects of isotropization in the noncommutative minisuperspace seems to disappear. In the quantum regimen, the probability density presents a new structure that corresponds to the value of the noncommutativity parameter.
\end{abstract}

PACS numbers: 02.40.Gh,04.60.Kz,98.80.Qc

\section{INTRODUCTION}

The inclusion of matter into the homogeneous cosmologies is usually done with the inclusion of scalar fields, in order to study different scenarios, such as inflation, dark matter, dark energy. However, since the early seventies, the problem of the appropriate sources of matter to solve an specific scenario [1, 2], and its corresponding lagrangian exists. In this paper we consider the scalar field and perfect fluid sources as a first approximation in the noncommutative quantization regime, and in particular for the Bianchi type I cosmological model, which is the generalization of the flat FRW cosmological model. Bianchi cosmological models are the homogeneous but anisotropic generalization of the FRW cosmological model, in which the isotropy is not required. In view of the present data that implies a high degree isotropy in the Universe, the Bianchi model need an isotropization mechanism to agree with the observation. Otherwise they can only model earlier stages of the Universe. In the past different kinds o matter have been used to obtain the isotropization. In this paper we explore another possibility to aquire the isotropization in the cosmological model: that noncommutativity among the material content (fields and perfect fluid) and the geometric variables of the cosmological models produces the expected isotropy. We will explore the effects of noncommutativity in the classical and quantum regimes.

In the last years there has been several attempts to study the possible effects of noncommutativity in the cosmological scenario. In particular, in [4], the authors in a cunning way avoided the difficult technicalities of analyzing

\footnotetext{
*Electronic address: socorro@fisica.ugto.mx

†Electronic address: lopr@xanum.uam.mx

$\ddagger$ Electronic address: ortizgca@fisica.uaz.edu.mx

$\S$ Electronic address: makxim@gmail.com
} 
noncommutative cosmological models, when these are derived from a noncommutative theory of gravity $[5]$. Their proposal is to introduce the effects of noncommutativity in quantum cosmology, by a deformation of the minisuperspace, and it is achieved by means of a Moyal deformation of the WDW equation, similar to the case of the noncommutative quantum mechanics [6, 7]. Some work has been done in this direction, for example in [8] the authors study the implications of noncommutative geometry in minisuperspace variables for an FRW universe with a conformally coupled scalar field, using the Bohmian formalism of quantum trajectories [9], also in [10] a noncommutative deformation with a scalar field in the scalar-tensor theory of gravity was considered. Recently, some work on noncommutative fields has appeared, see for example Carmona et al. [11], where the authors gave the generalization to the noncommutative harmonic oscillator and have shown the most general transformation between the coordinates and momenta. In Vakili et al. [12] a similar procedure is applied to Bianchi I models without matter, and in [13] the matter is included.

In order to obtein a deformed model, there are several ways to proceed, such as [14], where the uncentainty relations are generalized, which can be of interest, in order to obtain new information in the cosmological scenario and other physical quantities. An alternative method that consider deformed special relativity, is considered in [15], where the authors study the effects of the existence of a fundamental length in a cosmological scenario, this is described by what is known as the $\kappa$-deformation [16]. Both approaches have the same role, to obtain fundamental length, because is generally believe that is a natural feature in all theories which have the duty to obtain the quantization of gravity.

The aim of this paper is to construct a noncommutative scenario for the Bianchi class A cosmological models, introducing noncommutative scale factors, considering that all minisuperspace variables $\mathrm{q}^{\mathrm{i}}$ do not commute, so the symplectic structure is modified, in order to study the consequences of the noncommutative in the universe, such as the isotropization of the anisotropic parameters $\beta_{ \pm}$, so the decay of the field $\phi$ dynamically. The other methods could be implemented and carry the analysis if those methods give a better dynamical isotropization of the anisotropic parameters. This will be reported in future work.

The paper is then organized as follows. In Sec. II, we obtain the Wheeler-DeWitt equation including several matter contribution, a scalar field, a barotropic perfect fluid and a cosmological term. Also we obtain the corresponding classical solutions. In Sec. III we present the commutative quantum solutions for the cosmological Bianchi type I, considering various scenarios, including the effect to the $\Lambda$ cosmological term. In Sec. IV and V, the corresponding non commutative classical and quantum solutions are presented, taking that all the minisuperspace variables do not commute. Final remarks, are given in Sec. VI.

\section{THE WHEELER-DEWITT EQUATION}

Recalling the canonical formulation of the ADM formalism of the diagonal Bianchi Class A cosmological models. The metrics have the form

$$
\mathrm{ds}^{2}=-\left(\mathrm{N}^{2}-\mathrm{N}^{\mathrm{j}} \mathrm{N}_{\mathrm{j}}\right) \mathrm{dt}^{2}+\mathrm{e}^{2 \Omega(\mathrm{t})} \mathrm{e}^{2 \beta_{\mathrm{ij}}(\mathrm{t})} \omega^{\mathrm{i}} \omega^{\mathrm{j}}
$$

where $\mathrm{N}$ and $\mathrm{N}_{\mathrm{i}}$ are the lapse and shift functions, respectively, $\Omega(t)$ is a scalar and $\beta_{\mathrm{ij}}(\mathrm{t})$ a $3 \mathrm{x} 3$ diagonal matrix, $\beta_{\mathrm{ij}}=\operatorname{diag}\left(\beta_{+}+\sqrt{3} \beta_{-}, \beta_{+}-\sqrt{3} \beta_{-},-2 \beta_{+}\right), \omega^{\mathrm{i}}$ are one-forms that characterize each cosmological Bianchi type model, that obey $\mathrm{d} \omega^{\mathrm{i}}=\frac{1}{2} \mathrm{C}_{\mathrm{jk}}^{\mathrm{i}} \omega^{\mathrm{j}} \wedge \omega^{\mathrm{k}}, \mathrm{C}_{\mathrm{jk}}^{\mathrm{i}}$ the structure constants of the corresponding invariance group [2]. The metric for the Bianchi type I, takes the form

$$
\mathrm{ds}_{\mathrm{I}}^{2}=-\mathrm{N}^{2} \mathrm{dt}^{2}+\mathrm{e}^{2 \Omega} \mathrm{e}^{2 \beta_{+}+2 \sqrt{3} \beta_{-}} \mathrm{dx}^{2}+\mathrm{e}^{2 \Omega} \mathrm{e}^{2 \beta_{+}-2 \sqrt{3} \beta_{-}} \mathrm{dy}^{2}+\mathrm{e}^{2 \Omega} \mathrm{e}^{-4 \beta_{+}} \mathrm{dz}^{2}
$$


The Lagrangian function is given by

$$
\mathrm{L}_{\text {Total }}=\sqrt{-\mathrm{g}}(\mathrm{R}-2 \Lambda)+\mathrm{L}_{\text {matter }}
$$

we use as a first approximation a perfect fluid and a scalar field as the matter content, in a comoving frame [2, 17],

$$
\mathrm{L}_{\text {Total }}=\sqrt{-\mathrm{g}}\left(\mathrm{R}-2 \Lambda+16 \pi \mathrm{G} \rho+\frac{1}{2} \mathrm{~g}^{\mu \nu} \partial_{\mu} \phi \partial_{\nu} \phi+\mathrm{V}(\phi)\right)
$$

and using (2), we obtain the corresponding Hamiltonian function

$$
\mathrm{H}=\frac{\mathrm{e}^{-3 \Omega}}{24}\left[-\mathrm{P}_{\Omega}^{2}+\mathrm{P}_{+}^{2}+\mathrm{P}_{-}^{2}+12 \mathrm{P}_{\phi}^{2}-48 \Lambda \mathrm{e}^{6 \Omega}+384 \pi \mathrm{GM}_{\gamma} \mathrm{e}^{-3(\gamma-1) \Omega}+12 \mathrm{e}^{6 \Omega} \mathrm{V}(\phi)\right]
$$

together with barotropic state equation $\mathrm{p}=\gamma \rho$. The WDW equation for these models is obtained by the usual identification, $\mathrm{P}_{\mathrm{q}^{\mu}}$ by $-\mathrm{i} \partial_{\mathrm{q}^{\mu}}$ in (5), with $\mathrm{q}^{\mu}=\left(\Omega, \beta_{+}, \beta_{-}, \phi\right)$.

Following Hartle and Hawking [18], we consider a semi-general factor ordering which gives

$$
\mathrm{e}^{-3 \Omega}\left[\frac{\partial^{2}}{\partial \Omega^{2}}-\frac{\partial^{2}}{\partial \beta_{+}^{2}}-\frac{\partial^{2}}{\partial \beta_{-}^{2}}-12 \frac{\partial^{2}}{\partial \phi^{2}}+\mathrm{Q} \frac{\partial}{\partial \Omega}-48 \Lambda \mathrm{e}^{6 \Omega}+384 \pi \mathrm{GM}_{\gamma} \mathrm{e}^{-3(\gamma-1) \Omega}+12 \mathrm{e}^{6 \Omega} \mathrm{V}(\phi)\right] \Psi=0
$$

This equation is not easy to solve in general, therefore we consider several particular cases that yield analytical solutions, always considering the scalar field.

\section{A. Classical Solutions via Hamiltonian Formalism}

To find the commutative equation of motion, we use the classical phase space variables $\mathrm{q}^{\mu}=\left(\Omega, \beta_{ \pm}, \phi\right)$, where the Poisson algebra for these minisuperspace variables are

$$
\left\{\mathrm{q}^{\mu}, \mathrm{q}^{\nu}\right\}=0 \quad\left\{\mathrm{q}^{\mu}, \mathrm{P}_{\mathrm{q}^{\nu}}\right\}=\delta_{\nu}^{\mu}
$$

and recalling the Hamiltonian equation (5), we obtain the classical solutions.

The classical equations of motion for the phase variables $q^{\mu}$ and $P_{q^{\nu}}$ are

$$
\begin{aligned}
\dot{\Omega}= & \{\Omega, \mathrm{H}\}=-\frac{1}{12} \mathrm{e}^{-3 \Omega} \mathrm{P}_{\Omega}, \\
\dot{\beta}_{-}= & \left\{\beta_{-}, \mathrm{H}\right\}=\frac{1}{12} \mathrm{e}^{-3 \Omega} \mathrm{P}_{-}, \\
\dot{\beta}_{+}= & \left\{\beta_{+}, \mathrm{H}\right\}=\frac{1}{12} \mathrm{e}^{-3 \Omega} \mathrm{P}_{+}, \\
\dot{\phi}= & \{\phi, \mathrm{H}\}=\mathrm{e}^{-3 \Omega} \mathrm{P}_{\phi}, \\
\dot{\mathrm{P}}_{\Omega}= & \left\{\mathrm{P}_{\Omega}, \mathrm{H}\right\}=\frac{1}{8} \mathrm{e}^{-3 \Omega}\left[-\mathrm{P}_{\Omega}^{2}+\mathrm{P}_{-}^{2}+\mathrm{P}_{+}^{2}+12 \mathrm{P}_{\phi}^{2}\right] \\
& +\left[48 \Lambda e^{6 \Omega}+\gamma b_{\gamma} e^{-3(\gamma-1) \Omega}-12 e^{6 \Omega} V(\phi)\right], \\
\dot{\mathrm{P}}_{-}= & \left\{\mathrm{P}_{-}, \mathrm{H}\right\}=0, \quad \rightarrow \mathrm{P}_{-}= \pm \mathrm{p}_{1}=\text { const. } \\
\dot{\mathrm{P}}_{+}= & \{\phi, \mathrm{H}\}=, \quad \mathrm{P}_{+}= \pm \mathrm{n}_{1}=\text { const. } \\
\dot{\mathrm{P}}_{\phi}= & \left\{\mathrm{P}_{+}, \mathrm{H}\right\}=\frac{1}{8} \mathrm{e}^{-3 \Omega} \frac{\mathrm{dV}(\phi)}{\mathrm{d} \phi} .
\end{aligned}
$$

In order to have an analytic solution, we have consider different cases

$$
V(\phi)=V_{0}=\text { cte. } \rightarrow P_{\phi}=\text { const }=\frac{m_{1}}{\sqrt{12}}
$$


we choose the constant $P_{\phi}$ in such way for simplicity. In this case, we can associate the potential field with the cosmological term, so we have an effective parameter $\lambda_{\text {eff }}=48 \Lambda-12 V_{0}$ for $\gamma \neq-1$, and $\lambda_{\text {eff }}=48 \Lambda-384 \pi G M_{-1}-$ $12 V_{0}$ for the case $\gamma=-1$. We obtain the relation for $\mathrm{P}_{\Omega}$

$$
\mathrm{P}_{\Omega}= \pm \sqrt{\mathrm{a}_{1}^{2}-\lambda_{\text {eff }} \mathrm{e}^{6 \Omega}+\mathrm{b}_{\gamma} \mathrm{e}^{-3(\gamma-1) \Omega}}
$$

where $\mathrm{a}_{1}^{2}=\mathrm{n}_{1}^{2}+\mathrm{p}_{1}^{2}+\mathrm{m}_{1}^{2}$. Employing this relation together with Eq.(8), for particular epochs of the universe evolution, characterized by different values of the $\gamma$ parameter, we present the classical solutions in table IA.

\begin{tabular}{|c|c|}
\hline Case & Commutative solutions \\
\hline $\begin{array}{l}\gamma=-1, \quad \lambda_{\text {eff }} \neq 0, \quad \rho_{-1}=\mathrm{M}_{-1} \\
\mathrm{q}^{2}=-\frac{\lambda_{\text {eff }}}{16} \\
\mathrm{a}_{1}^{2}=\mathrm{n}_{1}^{2}+\mathrm{p}_{1}^{2}+\mathrm{m}_{1}^{2},\end{array}$ & $\begin{array}{l}\Omega=\frac{1}{3} \operatorname{Ln}\left[\frac{\mathrm{e}^{2 \mathrm{qt}}-4 \mathrm{a}_{1}^{2}}{16 \mathrm{qe}^{\mathrm{qt}}}\right] \\
\beta_{+}= \pm \frac{2}{3} \frac{\mathrm{n}_{1}}{\mathrm{a}_{1}} \operatorname{arctanh}\left[\frac{\mathrm{e}^{\mathrm{qt}}}{2 \mathrm{a}_{1}}\right] \\
\beta_{-}= \pm \frac{2}{3} \frac{\mathrm{p}_{1}}{\mathrm{a}_{1}} \operatorname{arctanh}\left[\frac{\mathrm{e}^{\mathrm{qt}}}{2 \mathrm{a}_{1}}\right] \\
\phi= \pm \frac{4}{\sqrt{6}} \frac{\mathrm{m}_{1}}{\mathrm{a}_{1}} \operatorname{arctanh}\left[\frac{\mathrm{e}^{\mathrm{qt}}}{2 \mathrm{a}_{1}}\right.\end{array}$ \\
\hline $\begin{array}{l}\gamma=1, \quad \lambda_{e f f}<0, \quad \rho_{1}=\mathrm{M}_{1} \mathrm{e}^{-6 \Omega} \\
\mathrm{q}=\sqrt{3\left|\Lambda-\frac{\mathrm{V}_{0}}{4}\right|}, \\
\mathrm{a}_{1}^{2}=\mathrm{n}_{1}^{2}+\mathrm{p}_{1}^{2}+\mathrm{m}_{1}^{2}+384 \pi \mathrm{GM}_{1},\end{array}$ & $\begin{array}{l}\Omega=\frac{1}{3} \operatorname{Ln}\left[\frac{\mathrm{e}^{2 \mathrm{qt}}-4 \mathrm{a}_{1}^{2}}{16 \mathrm{qe}^{\mathrm{qt}}}\right] \\
\beta_{+}= \pm \frac{2}{3} \frac{\mathrm{n}_{1}}{\mathrm{a}_{1}} \operatorname{arctanh}\left[\frac{\mathrm{e}^{\mathrm{qt}}}{2 \mathrm{a}_{1}}\right] \\
\beta_{-}= \pm \frac{2}{3} \frac{\mathrm{p}_{1}}{\mathrm{a}_{1}} \operatorname{arctanh}\left[\frac{\mathrm{e}^{\mathrm{qt}}}{2 \mathrm{a}_{1}}\right] \\
\phi= \pm \frac{4}{\sqrt{6}} \frac{\mathrm{m}_{1}}{\mathrm{a}_{1}} \operatorname{arctanh}\left[\frac{\mathrm{e}^{\mathrm{qt}}}{2 \mathrm{a}_{1}}\right.\end{array}$ \\
\hline $\begin{array}{l}\gamma=1, \quad \lambda_{\text {eff }}=0, \quad \rho_{1}=\mathrm{M}_{1} \mathrm{e}^{-6 \Omega} \\
\mathrm{a}_{1}^{2}=\mathrm{n}_{1}^{2}+\mathrm{p}_{1}^{2}+\mathrm{m}_{1}^{2}+384 \pi \mathrm{GM}_{1},\end{array}$ & $\begin{array}{l}\Omega=\frac{1}{3} \operatorname{Ln}\left[\frac{\mathrm{a}_{1}}{4} \mathrm{t}\right] \\
\beta_{+}= \pm \operatorname{Ln}\left[\mathrm{t}^{-\frac{\mathrm{n}_{1}}{3 \mathrm{a}_{1}}}\right] \\
\beta_{-}= \pm \operatorname{Ln}\left[\mathrm{t}^{-\frac{\mathrm{p}_{1}}{3 \mathrm{a}_{1}}}\right] \\
\phi= \pm \operatorname{Ln}\left[\mathrm{t}^{-\frac{2 \mathrm{~m}_{1}}{\sqrt{6} \mathrm{a}_{1}}}\right]\end{array}$ \\
\hline $\begin{array}{l}\gamma=0, \quad \lambda_{e f f}=0, \quad \rho_{0}=\mathrm{M}_{0} \mathrm{e}^{-3 \Omega} \\
\mathrm{b}_{0}=384 \pi \mathrm{GM}_{0}, \\
\mathrm{a}_{1}^{2}=\mathrm{n}_{1}^{2}+\mathrm{p}_{1}^{2}+\mathrm{m}_{1}^{2},\end{array}$ & $\begin{array}{l}\Omega=\frac{1}{3} \operatorname{Ln}\left[\frac{\mathrm{b}_{0} \mathrm{t}^{2}}{64}+\frac{\mathrm{a}_{1} \mathrm{t}}{4}\right] \\
\beta_{+}= \pm \frac{\mathrm{n}_{1}}{3 \mathrm{a}_{1}} \operatorname{Ln}\left[\frac{16 \mathrm{a}_{1}+\mathrm{b}_{0} \mathrm{t}}{\mathrm{t}}\right] \\
\beta_{-}= \pm \frac{\mathrm{p}_{1}}{3 \mathrm{a}_{1}} \operatorname{Ln}\left[\frac{16 \mathrm{a}_{1}+\mathrm{b}_{0} \mathrm{t}}{\mathrm{t}}\right] \\
\phi= \pm \frac{4 \mathrm{~m}_{1}}{\sqrt{6} \mathrm{a}_{1}} \operatorname{Ln}\left[\frac{16 \mathrm{a}_{1}+\mathrm{b}_{0} \mathrm{t}}{\mathrm{t}}\right]\end{array}$ \\
\hline
\end{tabular}

In all cases $\beta_{ \pm}$are not bounded, excluding isotropization. In the present work we will find out how the noncommutativity solve this problem.

\section{COMMUTATIVE QUANTUM SOLUTION}

In the following we consider different cases of the potential that allowed us to obtain exact solutions.

$$
\text { A. } \mathrm{V}(\phi)=\mathrm{V}_{0}
$$

For this case, the potential field is equivalent to a cosmological term, and it is included in the $\Lambda_{\text {eff }}$ parameter. Using the method of separation of variables, 
$\Psi\left(\Omega, \beta_{ \pm}, \phi\right)=\mathcal{A}(\Omega) \mathcal{B}\left(\beta_{+}\right) \mathcal{C}\left(\beta_{-}\right) \mathcal{D}(\phi)$, in Eq. (6) we find

$$
\begin{aligned}
\frac{\mathrm{d}^{2} \mathcal{A}}{\mathrm{d} \Omega^{2}}+\mathrm{Q} \frac{\mathrm{d} \mathcal{A}}{\mathrm{d} \Omega}+\left(384 \pi \mathrm{GM}_{\gamma} \mathrm{e}^{-3(\gamma-1) \Omega}+\Lambda_{\mathrm{eff}} \mathrm{e}^{6 \Omega}+\alpha^{2}\right) \mathcal{A} & =0 \\
\frac{\mathrm{d}^{2} \mathcal{B}}{\mathrm{d} \beta_{+}^{2}}+\nu^{2} \mathcal{B} & =0 \\
\frac{\mathrm{d}^{2} \mathcal{C}}{\mathrm{d} \beta_{-}^{2}}+\mu^{2} \mathcal{C} & =0 \\
\frac{\mathrm{d}^{2} \mathcal{D}}{\mathrm{d} \phi^{2}}+\eta^{2} \mathcal{D} & =0
\end{aligned}
$$

where $\Lambda_{\text {eff }}=+12 \mathrm{~V}_{0}-48 \Lambda$ is the effective cosmological constant now, and the separation constants are related by $\mu^{2}=\alpha^{2}-\nu^{2}-12 \eta^{2}$. The solutions to (19, 201) and (21) are

$$
\begin{aligned}
\mathcal{B} & =\mathrm{A}_{1} \mathrm{e}^{\mathrm{i} \nu \beta_{+}}+\mathrm{B}_{1} \mathrm{e}^{-\mathrm{i} \nu \beta_{+}}, \\
\mathcal{C} & =\mathrm{A}_{2} \mathrm{e}^{\mathrm{i} \mu \beta_{-}}+\mathrm{B}_{2} \mathrm{e}^{-\mathrm{i} \mu \beta_{-}}, \\
\mathcal{D} & =\mathrm{A}_{3} \mathrm{e}^{\mathrm{i} \eta \phi}+\mathrm{B}_{3} \mathrm{e}^{-\mathrm{i} \eta \phi},
\end{aligned}
$$

where $A_{j}, B_{j}, j=1,2,3$ are integration constants.

The general solution to equation (18) is very complicated to find, so in the following we will solve particular for particular cases.

$$
\text { 1. } \Lambda_{\mathrm{eff}}=0, \gamma \neq 1 \text {, and factor ordering } \mathrm{Q} \neq 0 \text {. }
$$

For this choice of the parameters, the equation (18) is

$$
\frac{\mathrm{d}^{2} \mathcal{A}}{\mathrm{d} \Omega^{2}}+\mathrm{Q} \frac{\mathrm{d} \mathcal{A}}{\mathrm{d} \Omega}+\left(384 \pi \mathrm{GM}_{\gamma} \mathrm{e}^{-3(\gamma-1) \Omega}+\alpha^{2}\right) \mathcal{A}=0
$$

this equation can be written as an ordinary Bessel equation by making the transformation $\mathrm{z}=\mathrm{z}_{0} \mathrm{e}^{-3(\gamma-1) \Omega}, \mathrm{z}_{0}=$ $384 \pi \mathrm{GM}_{\gamma}$,

$$
\mathrm{z}^{2} \frac{\mathrm{d}^{2} \mathcal{A}}{\mathrm{dz}^{2}}+\left(1+\frac{\mathrm{Q}}{9(\gamma-1)^{2}}\right) \mathrm{z} \frac{\mathrm{d} \mathcal{A}}{\mathrm{dz}}+\frac{1}{9(\gamma-1)^{2}}\left(\mathrm{z}+\alpha^{2}\right) \mathcal{A}=0
$$

whose solution becomes

$$
\mathcal{A}_{\rho}=\mathrm{z}^{\mathrm{q}_{0}} \mathrm{~J}_{ \pm \mathrm{i} \rho}\left(\frac{2}{3(\gamma-1)} \mathrm{z}^{\frac{1}{2}}\right), \quad \mathrm{q}_{0}=-\frac{\mathrm{Q}}{18(\gamma-1)^{2}}
$$

where $\rho=\frac{1}{3(\gamma-1)} \sqrt{4 \alpha^{2}-\left(\frac{\mathrm{Q}}{3(\gamma-1)}\right)^{2}}$, with $\pm \mathrm{i} \rho$ the order of the ordinary Bessel function.

Then, we find For the complete wave function, we find the equation

$$
\begin{aligned}
& \Psi\left(\Omega, \beta_{ \pm}, \phi\right)=\mathrm{z}^{\mathrm{q} 0} \mathrm{~J}_{ \pm \mathrm{i} \rho}\left(\frac{2 \mathrm{z}^{\frac{1}{2}}}{3(\gamma-1)}\right)\left[\mathrm{A}_{3} \mathrm{e}^{\mathrm{i} \eta \phi}+\mathrm{B}_{3} \mathrm{e}^{-\mathrm{i} \eta \phi}\right] \\
& \left(\mathrm{A}_{1} \mathrm{e}^{\mathrm{i} \nu \beta_{+}}+\mathrm{B}_{1} \mathrm{e}^{-\mathrm{i} \nu \beta_{+}}\right)\left[\mathrm{A}_{2} \mathrm{e}^{\mathrm{i} \mu \beta_{-}}+\mathrm{B}_{2} \mathrm{e}^{-\mathrm{i} \mu \beta_{-}}\right] .
\end{aligned}
$$

To obtain the complete wavepacket out of the wavefunction is complicated, that is because all parameters are interrelated, so we limit the study to build 3D wavepacket by considering two variables field. This is carry out for the case where the parameter $\gamma$ take the value -1 (inflation phenomenon). 


\section{2. $\Lambda_{\text {eff }} \neq 0, \gamma=1$, factor ordering $\mathrm{Q} \neq 0$.}

When we introduce the effective cosmological constant $\Lambda_{\text {eff }}$ and a stiff fluid, equation (18) takes the following form

$$
\frac{\mathrm{d}^{2} \mathcal{A}}{\mathrm{d} \Omega^{2}}+\mathrm{Q} \frac{\mathrm{d} \mathcal{A}}{\mathrm{d} \Omega}+\left(\Lambda_{\text {eff }} \mathrm{e}^{6 \Omega}+\mathrm{c}_{1}\right) \mathcal{A}=0
$$

where $\mathrm{c}_{1}=384 \pi \mathrm{GM}_{1}+\alpha^{2}$, and making the transformation $\mathrm{z}=\Lambda_{\text {eff }} \mathrm{e}^{6 \Omega}$, we find the solutions in terms of generic Bessel functions

$$
\begin{aligned}
& \mathcal{A}_{\rho}(\mathrm{z})=\mathrm{z}^{-\frac{\mathrm{Q}}{12}} \mathrm{Z}_{\rho}\left( \pm \frac{1}{3} \mathrm{z}^{\frac{1}{2}}\right), \\
& \mathcal{A}_{\rho}(\Omega)=\mathrm{A}_{0} \mathrm{e}^{-\frac{\mathrm{Q}}{2} \Omega} \mathrm{Z}_{\rho}\left( \pm \frac{1}{3} \sqrt{\lambda}^{3 \Omega}\right) .
\end{aligned}
$$

with order $\rho=\frac{1}{6} \sqrt{\mathrm{Q}^{2}-4 \mathrm{c}_{1}}$ and $\mathrm{Z}_{\rho}$ is a generic Bessel function.

$$
\text { 3. } \Lambda_{\text {eff }} \neq 0, \gamma=-1 \text {, factor ordering } \mathrm{Q} \neq 0
$$

The other interesting case, corresponds to $\gamma=-1$ (the equation of state for inflation), proceeding as before, we make the transformation $\mathrm{z}=\mathrm{be}^{6 \Omega}$, where $\mathrm{b}=384 \pi \mathrm{GM}_{-1}+\Lambda$, we arrive to equation

$$
\mathrm{z}^{2} \frac{\mathrm{d}^{2} \mathcal{A}}{\mathrm{dz}^{2}}+\left(1+\frac{\mathrm{Q}}{6}\right) \mathrm{z} \frac{\mathrm{d} \mathcal{A}}{\mathrm{dz}}+\frac{1}{36}\left(\mathrm{z}+\alpha^{2}\right) \mathcal{A}=0
$$

the solution is written with the generic Bessel functions

$$
\mathcal{A}(\mathrm{z})=\mathrm{z}^{-\frac{Q}{12}} \mathrm{Z}_{ \pm \mathrm{i} \rho}\left( \pm \frac{1}{3} \mathrm{z}^{\frac{1}{2}}\right)
$$

with order $\rho=\frac{1}{6} \sqrt{4 \alpha^{2}-\mathrm{Q}^{2}}$.

From equations $(22,23,24)$ we notice that the dependence of the wavefunction on the variables $\phi$ and $\beta_{ \pm}$have the same structure. In the aim of building a Gaussian weighted wavepacket [20, 21], we fix the attention to solutions in two of the four variables. For simplicity we choose $\Omega$ and the scalar field $\phi$. We also choose the particular case in which the factor ordering $Q$ is zero,

$$
\Psi(\Omega, \phi)=\mathcal{N} \int_{-\infty}^{\infty} \mathrm{e}^{-\mathrm{a}(\rho-\mathrm{b})^{2}} \mathrm{e}^{-\frac{\sqrt{3}}{2} \rho \phi} \mathrm{J}_{\mathrm{i} \rho}\left(\frac{1}{3} \sqrt{\mathrm{b}} \mathrm{e}^{3 \Omega}\right) \mathrm{d} \rho,
$$

the corresponding plot is presented in figure (1), taking the particular values for $a=1.5$ and $b=1.3$; and as in the [4, 14] the integral was performed numerically. In this plot the wave function is not peaked around any particular values of the variables $(\Omega, \phi)$.

$$
\text { B. } \mathrm{V}(\phi)=\mathrm{V}_{0} \mathrm{e}^{-\beta \phi} \text {, stiff fluid } \gamma=1 \text { and } \Lambda=0
$$

The equation (6) becomes

$$
\left[\frac{\partial^{2}}{\partial \Omega^{2}}-\frac{\partial^{2}}{\partial \beta_{+}^{2}}-\frac{\partial^{2}}{\partial \beta_{-}^{2}}-12 \frac{\partial^{2}}{\partial \phi^{2}}+\mathrm{Q} \frac{\partial}{\partial \Omega}+384 \pi \mathrm{GM}_{1}+12 \mathrm{~V}_{0} \mathrm{e}^{6 \Omega-\beta \phi}\right] \Psi=0
$$




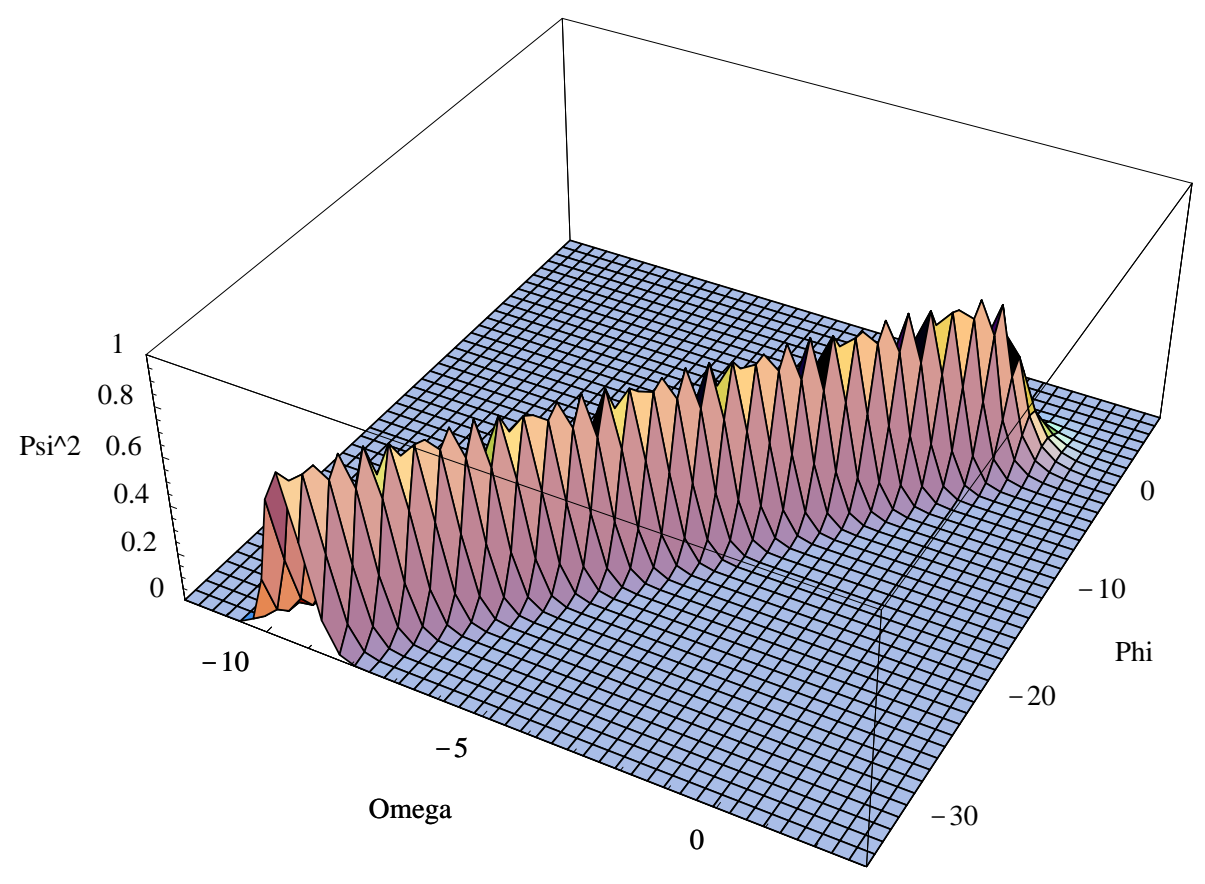

FIG. 1: 3D plot $|\Psi|^{2}$ corresponding to the Gaussian wavepacket equation (34) in the commuting case, with $\mu=\nu=0$

using the ansatz, $\Psi\left(\Omega, \beta_{ \pm}, \phi\right)=\mathcal{F}(\Omega, \phi) \mathcal{B}\left(\beta_{+}\right) \mathcal{C}\left(\beta_{-}\right)$, on Eq. (35) we find

$$
\begin{gathered}
\frac{1}{\mathcal{F}} \frac{\partial^{2} \mathcal{F}}{\partial \Omega^{2}}+\frac{\mathrm{Q}}{\mathcal{F}} \frac{\partial \mathcal{F}}{\partial \Omega}-\frac{12}{\mathcal{F}} \frac{\partial^{2} \mathcal{F}}{\partial \phi^{2}}+12 \mathrm{~V}_{0} \mathrm{e}^{6 \Omega-\beta \phi}+\mathrm{c}=0, \\
\frac{\mathrm{d}^{2} \mathcal{B}}{\mathrm{d} \beta_{+}^{2}}+\nu^{2} \mathcal{B}=0, \frac{\mathrm{d}^{2} \mathcal{C}}{\mathrm{d} \beta_{-}^{2}}+\mu^{2} \mathcal{C}=0,
\end{gathered}
$$

where $\mathrm{c}=\alpha^{2}+384 \pi \mathrm{GM}_{1}$, and the constants satisfy the relation $\mu^{2}=\alpha^{2}-\nu^{2}$.

There are many changes of variables for equation (36) that allows to solve it by separation of variables, we choose the following one

$$
\mathrm{x}=-6 \Omega+\beta \phi, \quad \mathrm{y}=-2 \Omega+\frac{\phi}{\beta},
$$

which is valid for $\beta^{2} \neq 3$, then equation (36), using $\mathcal{F}=X(x) Y(y)$, is transformed into the following two equations

$$
\begin{aligned}
& \frac{\mathrm{d}^{2} \mathrm{X}}{\mathrm{dx^{2 }}}-\frac{\mathrm{Q}}{2\left(3-\beta^{2}\right)} \frac{\mathrm{dX}}{\mathrm{dx}}++\frac{1}{12\left(3-\beta^{2}\right)}\left[12 \mathrm{~V}_{0} \mathrm{e}^{-\mathrm{x}}+\mathrm{n}^{2}\right] \mathrm{X}=0, \\
& \frac{\mathrm{d}^{2} \mathrm{Y}}{\mathrm{dy}^{2}}+\frac{2 \mathrm{Q} \beta^{2}}{4\left(3-\beta^{2}\right)} \frac{\mathrm{d} \mathrm{Y}}{\mathrm{dy}}-\frac{\beta^{2} \mathrm{~m}^{2}}{4\left(3-\beta^{2}\right)} \mathrm{Y}=0,
\end{aligned}
$$

with $\mathrm{n}^{2}=\mathrm{c}-\mathrm{m}^{2}$. Substituting $\mathrm{z}=\mathrm{e}^{-\mathrm{x}}$ in (39), we obtain a Bessel differential equation whose solution is

$$
\mathrm{X}(\mathrm{x})=\operatorname{Exp}\left[-\frac{\mathrm{Q}}{4\left(3-\beta^{2}\right)} \mathrm{x}\right] \mathrm{J}_{ \pm \mathrm{r}}\left(\sqrt{\frac{\mathrm{V}_{0}}{3-\beta^{2}}} \mathrm{e}^{-\mathrm{x} / 2}\right)
$$

with $r=\sqrt{\frac{\mathrm{Q}^{2}}{4\left(3-\beta^{2}\right)^{2}}-\frac{\mathrm{n}^{2}}{3\left(3-\beta^{2}\right)}}$, the corresponding order. The other equation has the solution

$$
\mathrm{Y}(\mathrm{y})=\mathrm{e}^{\mathrm{sy}}\left[\mathrm{A}_{4} \mathrm{e}^{\frac{\mathrm{Q} \beta^{2}}{4\left(3-\beta^{2}\right)} \mathrm{y}}+\mathrm{B}_{4} \mathrm{e}^{-\frac{\mathrm{Q} \beta^{2}}{4\left(3-\beta^{2}\right)} \mathrm{y}}\right]
$$


with $\mathrm{s}=\sqrt{\left(\frac{\mathrm{Q} \beta^{2}}{4\left(3-\beta^{2}\right)}\right)^{2}+\frac{\mathrm{m}^{2} \beta^{2}}{4\left(3-\beta^{2}\right)}}$.

For the remaining case, $\beta^{2}=3$ the solution to the differential equation is

$$
\mathcal{F}=F_{1} e^{-\frac{Q \Omega}{2}}\left[e^{\frac{6 b x+a e^{6 x}}{6 \kappa}} e^{-\frac{\kappa y}{4}}\right],
$$

here $\kappa$ is a non null separation constant, $F_{1}$ is an arbitrary integration constant, $a=c-Q^{2} / 4, b=12 V_{0}$, and the new variables $x$ and $y$ are

$$
x=\frac{6 \Omega \mp \sqrt{3} \phi}{6}, y=\frac{6 \Omega \pm \sqrt{3} \phi}{6} .
$$

\section{NONCOMMUTATIVE CLASSICAL SOLUTION}

In the commutative model we know that the solutions to Hamilton's equations are the same as in General Relativity. Now the natural extension is to consider the noncommutative version of our model, with the idea of noncommutative between the four variables $\left(\Omega_{\mathrm{nc}}, \beta_{ \pm \mathrm{nc}}, \phi\right)$, so we apply a deformation of the Poisson algebra. For this we start with the usual Hamiltonian (5), but the symplectic structure is modify as follow

$$
\begin{aligned}
\left\{\mathrm{P}_{\Omega}, \mathrm{P}_{ \pm}\right\}_{\star} & =\left\{\mathrm{P}_{+}, \mathrm{P}_{-}\right\}_{\star}=0, \quad\left\{\mathrm{q}^{\mu}, \mathrm{P}_{\left.\mathrm{q}^{\mu}\right\}_{\star}}=1,\right. \\
\left\{\Omega, \beta_{+}\right\}_{\star} & =\mathrm{i} \theta_{1}, \quad\left\{\Omega, \beta_{-}\right\}_{\star}=\mathrm{i} \theta_{2}, \quad\left\{\beta_{-}, \beta_{+}\right\}_{\star}=\mathrm{i} \theta_{3}, \\
\{\Omega, \phi\}_{\star} & =\mathrm{i} \theta_{4}, \quad\left\{\phi, \beta_{+}\right\}_{\star}=\mathrm{i} \theta_{5}, \quad\left\{\phi, \beta_{-}\right\}_{\star}=\mathrm{i} \theta_{6} .
\end{aligned}
$$

where the $\star$ is the Moyal product [19], and the Hamiltonian resulting is

$$
\mathrm{H}_{\mathrm{nc}}=\frac{\mathrm{Ne}^{-3 \Omega_{\mathrm{nc}}}}{24}\left[-\mathrm{P}_{\Omega}^{2}+\mathrm{P}_{+}^{2}+\mathrm{P}_{-}^{2}+12 \mathrm{P}_{\phi}^{2}-\lambda \mathrm{e}^{6 \Omega_{\mathrm{nc}}}+12 \mathrm{~V}(\phi) \mathrm{e}^{6 \Omega_{\mathrm{nc}}}+\mathrm{b}_{\gamma} \mathrm{e}^{-3(\gamma-1) \Omega_{\mathrm{nc}}}\right]=0,
$$

but the symplectic structure is the one that we know, the commutative one (7). There are two formalism to study the noncommutative equations of motion, for the first formalism that we exposed have the original variables, but with the modified symplectic structure,

$$
\begin{aligned}
q_{n c}^{\dot{\mu}} & =\left\{\mathrm{q}^{\mu}, \mathrm{H}\right\}_{\star}, \\
P_{n c}^{\dot{\mu}} & =\left\{\mathrm{P}^{\mu}, \mathrm{H}\right\}_{\star},
\end{aligned}
$$

and for the second formalism we use the shifted variables but with the original (commutative) symplectic structure

$$
\begin{aligned}
q_{n c}^{\dot{\mu}} & =\left\{\mathrm{q}_{\mathrm{nc}}^{\mu}, \mathrm{H}_{\mathrm{nc}}\right\} \\
P_{n c}^{\dot{\mu}} & =\left\{\mathrm{P}_{\mathrm{nc}}^{\mu}, \mathrm{H}_{\mathrm{nc}}\right\},
\end{aligned}
$$


in both approaches we have the same result. Therefore the equations of motion take the form

$$
\begin{aligned}
\dot{\Omega}_{\mathrm{nc}} & =\{\Omega, \mathrm{H}\}_{\star}=\left\{\Omega_{\mathrm{nc}}, \mathrm{H}_{\mathrm{nc}}\right\}=-\frac{\mathrm{e}^{-3 \Omega_{\mathrm{nc}}}}{12} \mathrm{P}_{\Omega}, \\
\dot{\beta}_{-\mathrm{nc}} & =\left\{\beta_{-}, \mathrm{H}\right\}_{\star}=\left\{\beta_{-\mathrm{nc}}, \mathrm{H}_{\mathrm{nc}}\right\}=\frac{\mathrm{e}^{-3 \Omega_{\mathrm{nc}}}}{12} \mathrm{P}_{-}+\frac{\theta_{2}}{2} \dot{\mathrm{P}}_{\Omega}-\frac{\theta_{6}}{2} \mathrm{P}_{\phi}, \\
\dot{\beta}_{+\mathrm{nc}} & =\left\{\beta_{+}, \mathrm{H}\right\}_{\star}=\left\{\beta_{+\mathrm{nc}}, \mathrm{H}_{\mathrm{nc}}\right\}=\frac{\mathrm{e}^{-3 \Omega_{\mathrm{nc}}}}{12} \mathrm{P}_{+}+\frac{\theta_{1}}{2} \dot{\mathrm{P}}_{\Omega}-\frac{\theta_{5}}{2} \mathrm{P}_{\phi}, \\
\dot{\phi}_{\mathrm{nc}} & =\{\phi, \mathrm{H}\}_{\star}=\left\{\phi_{+\mathrm{nc}}, \mathrm{H}_{\mathrm{nc}}\right\}=\mathrm{e}^{-3 \Omega_{\mathrm{nc}}} \mathrm{P}_{\phi}-\frac{\theta_{4}}{2} \mathrm{P}_{\Omega}+\frac{\theta_{5}}{2} \mathrm{P}_{+}+\frac{\theta_{6}}{2} \mathrm{P}_{-} \\
\dot{\mathrm{P}}_{\Omega} & =\left\{\mathrm{P}_{\Omega}, \mathrm{H}\right\}_{\star}=\left\{\mathrm{P}_{\Omega}, \mathrm{H}_{\mathrm{nc}}\right\}=\frac{\mathrm{e}^{-3 \Omega_{\mathrm{nc}}}}{8}\left[6 \lambda \mathrm{e}^{6 \Omega_{\mathrm{nc}}}+3(\gamma-1) \mathrm{b}_{\gamma} \mathrm{e}^{-3(\gamma-1) \Omega_{\mathrm{nc}}}\right], \\
\dot{\mathrm{P}}_{-} & =\left\{\mathrm{P}_{-}, \mathrm{H}\right\}_{\star}=\left\{\mathrm{P}_{-}, \mathrm{H}_{\mathrm{nc}}\right\}=0, \rightarrow \quad \rightarrow \quad \mathrm{P}_{-}=\mathrm{p}_{1}, \\
\dot{\mathrm{P}}_{+} & =\left\{\mathrm{P}_{+}, \mathrm{H}\right\}_{\star}=\left\{\mathrm{P}_{+}, \mathrm{H}_{\mathrm{nc}}\right\}=0, \rightarrow \quad \rightarrow \quad \mathrm{P}_{+}=\mathrm{n}_{1} . \\
\dot{\mathrm{P}}_{\phi} & =\left\{\mathrm{P}_{\phi}, \mathrm{H}\right\}_{\star}=\left\{\mathrm{P}_{\phi}, \mathrm{H}_{\mathrm{nc}}\right\}=0, \rightarrow \quad \mathrm{P}_{\phi}=\frac{\mathrm{m}_{1}}{\sqrt{12}} .
\end{aligned}
$$

\begin{tabular}{|c|c|}
\hline ase & Noncommutative Solutions \\
\hline $\begin{array}{l}\gamma=-1, \quad \lambda_{\text {eff }} \neq 0, \quad \rho_{-1}=\mathrm{M}_{-1} \\
\mathrm{a}_{1}^{2}=\mathrm{n}_{1}^{2}+\mathrm{p}_{1}^{2}+\mathrm{m}_{1}^{2} \\
\mathrm{q}^{2}=-\frac{\lambda_{\text {eff }}}{16}\end{array}$ & $\begin{array}{l}\Omega_{\mathrm{nc}}=\frac{1}{3} \operatorname{Ln}\left[\frac{\mathrm{e}^{2 \mathrm{qt}^{\mathrm{t}}}-4 \mathrm{a}_{1}^{2}}{16 \mathrm{q}^{\mathrm{qt}}}\right]-\frac{\theta_{1}}{2} \mathrm{p}_{1}-\frac{\theta_{2}}{2} \mathrm{n}_{1}+\frac{\theta_{4}}{2 \sqrt{12}} \mathrm{~m}_{1} \\
\beta_{+\mathrm{nc}}= \pm \frac{2}{3} \frac{\mathrm{n}_{1}}{\mathrm{a}_{1}} \operatorname{arctanh}\left[\frac{\mathrm{e}^{\mathrm{qt}}}{2 \mathrm{a}_{1}}\right]+\frac{\theta_{1}}{8}\left(\frac{\mathrm{e}^{\mathrm{qt}}}{4}+\mathrm{a}_{1}^{2} \mathrm{e}^{-\mathrm{qt}}\right)-\frac{\theta_{3}}{2} \mathrm{p}_{1}-\frac{\theta_{6}}{2 \sqrt{12}} \mathrm{~m}_{1} \\
\beta_{-\mathrm{nc}}= \pm \frac{2}{3} \frac{\mathrm{p}_{1}}{\mathrm{a}_{1}} \operatorname{arctanh}\left[\frac{\mathrm{e}^{\mathrm{qt}}}{2 \mathrm{a}_{1}}\right]+\frac{\theta_{2}}{8}\left(\frac{\mathrm{e}^{\mathrm{qt}}}{4}+\mathrm{a}_{1}^{2} \mathrm{e}^{-\mathrm{qt}}\right)+\frac{\theta_{3}}{2} \mathrm{n}_{1}-\frac{\theta_{5}}{2 \sqrt{12}} \mathrm{~m}_{1} \\
\phi_{\mathrm{nc}}= \pm \frac{4}{\sqrt{6}} \frac{\mathrm{m}_{1}}{\mathrm{a}_{1}} \operatorname{arctanh}\left[\frac{\mathrm{e}^{\mathrm{qt}}}{2 \mathrm{a}_{1}}\right]-\frac{\theta_{4}}{2}\left(\frac{\mathrm{e}^{\mathrm{qt}}}{4}+\mathrm{a}_{1}^{2} \mathrm{e}^{-\mathrm{qt}}\right)+\frac{\theta_{5}}{2} \mathrm{n}_{1}+\frac{\theta_{6}}{2} \mathrm{p}_{1}\end{array}$ \\
\hline $\begin{array}{l}\gamma=1, \quad \lambda_{\text {eff }}<0, \quad \rho_{1}=\mathrm{M}_{1} \mathrm{e}^{-6 \Omega} \\
\mathrm{a}_{1}^{2}=\mathrm{n}_{1}^{2}+\mathrm{p}_{1}^{2}+\mathrm{m}_{1}^{2}+384 \pi \mathrm{GM}_{1}, \\
\mathrm{q}=\sqrt{3\left|\Lambda-\frac{\mathrm{V}_{0}}{4}\right|}\end{array}$ & $\begin{array}{l}\Omega_{\mathrm{nc}}=\frac{1}{3} \operatorname{Ln}\left[\frac{\mathrm{e}^{2 \mathrm{qt}^{\mathrm{t}}}-4 \mathrm{a}_{1}^{2}}{16 \mathrm{qe}^{\mathrm{qt}}}\right]-\frac{\theta_{1}}{2} \mathrm{p}_{1}-\frac{\theta_{2}}{2} \mathrm{n}_{1}+\frac{\theta_{4}}{2} \frac{\mathrm{m}_{1}}{\sqrt{12}} \\
\beta_{+\mathrm{nc}}= \pm \frac{2}{3} \frac{\mathrm{n}_{1}}{\mathrm{a}_{1}} \operatorname{arctanh}\left[\frac{\mathrm{e}^{\mathrm{qt}}}{2 \mathrm{a}_{1}}\right]+\frac{\theta_{1}}{8}\left(\frac{\mathrm{e}^{\mathrm{qt}}}{4}+\mathrm{a}_{1}^{2} \mathrm{e}^{-\mathrm{qt}}\right)-\frac{\theta_{3}}{2} \mathrm{p}_{1}-\frac{\theta_{6}}{2 \sqrt{12}} \mathrm{~m}_{1} \\
\beta_{-\mathrm{nc}}= \pm \frac{2}{3} \frac{\mathrm{p}_{1}}{\mathrm{a}_{1}} \operatorname{arctanh}\left[\frac{\mathrm{e}^{\mathrm{qt}}}{2 \mathrm{a}_{1}}\right]+\frac{\theta_{2}}{8}\left(\frac{\mathrm{e}^{\mathrm{qt}}}{4}+\mathrm{a}_{1}^{2} \mathrm{e}^{-\mathrm{qt}}\right)+\frac{\theta_{3}}{2} \mathrm{n}_{1}-\frac{\theta_{5}}{2 \sqrt{12}} \mathrm{~m}_{1} \\
\phi_{\mathrm{nc}}= \pm \frac{4}{\sqrt{6}} \frac{\mathrm{m}_{1}}{\mathrm{a}_{1}} \operatorname{arctanh}\left[\frac{\mathrm{e}^{\mathrm{qt}}}{2 \mathrm{a}_{1}}\right]-\frac{\theta_{4}}{2}\left(\frac{\mathrm{e}^{\mathrm{qt}}}{4}+\mathrm{a}_{1}^{2} \mathrm{e}^{-\mathrm{qt}}\right)+\frac{\theta_{5}}{2} \mathrm{n}_{1}+\frac{\theta_{6}}{2} \mathrm{p}_{1}\end{array}$ \\
\hline $\begin{array}{l}\gamma=1, \quad \lambda_{\text {eff }}=0, \quad \rho_{1}=\mathrm{M}_{1} \mathrm{e}^{-6 \Omega} \\
\mathrm{a}_{1}^{2}=\mathrm{n}_{1}^{2}+\mathrm{p}_{1}^{2}+\mathrm{m}_{1}^{2}+384 \pi \mathrm{GM}_{1}\end{array}$ & $\begin{array}{l}\Omega_{\mathrm{nc}}=\frac{1}{3} \operatorname{Ln}\left[\frac{\mathrm{a}_{1}}{4} \mathrm{t}\right]-\frac{\theta_{1}}{2} \mathrm{p}_{1}-\frac{\theta_{2}}{2} \mathrm{n}_{1}+\frac{\theta_{4}}{2 \sqrt{12}} \mathrm{~m}_{1} \\
\beta_{+\mathrm{nc}}= \pm \operatorname{Ln}\left[\mathrm{t}^{-\frac{\mathrm{n}_{1}}{3 \mathrm{a}_{1}}}\right]+\frac{\theta_{1}}{2} \mathrm{a}_{1}-\frac{\theta_{3}}{2} \mathrm{p}_{1}-\frac{\theta_{6}}{2 \sqrt{12}} \mathrm{~m}_{1} \\
\beta_{-\mathrm{nc}}= \pm \operatorname{Ln}\left[\mathrm{t}^{-\frac{\mathrm{p}_{1}}{3 \mathrm{a}_{1}}}\right]+\frac{\theta_{2}}{2} \mathrm{a}_{1}+\frac{\theta_{3}}{2} \mathrm{n}_{1}-\frac{\theta_{5}}{2 \sqrt{12}} \mathrm{~m}_{1} \\
\phi= \pm \operatorname{Ln}\left[\mathrm{t}^{-\frac{2 \mathrm{~m}_{1}}{\sqrt{6} \mathrm{a}_{1}}}\right]-\frac{\theta_{4}}{2} \mathrm{a}_{1}+\frac{\theta_{5}}{2} \mathrm{n}_{1}+\frac{\theta_{6}}{2} \mathrm{p}_{1}\end{array}$ \\
\hline $\begin{array}{l}\gamma=0, \quad \lambda_{\text {eff }}=0, \quad \rho_{0}=\mathrm{M}_{0} \mathrm{e}^{-3 \Omega} \\
\mathrm{b}_{0}=384 \pi \mathrm{GM}_{0}, \\
\mathrm{a}_{1}^{2}=\mathrm{n}_{1}^{2}+\mathrm{p}_{1}^{2}+\mathrm{m}_{1}^{2},\end{array}$ & $\begin{array}{l}\Omega_{\mathrm{nc}}=\frac{1}{3} \operatorname{Ln}\left[\frac{\mathrm{b}_{0} \mathrm{t}^{2}}{64}+\frac{\mathrm{a}_{1} \mathrm{t}}{4}\right]-\frac{\theta_{1}}{2} \mathrm{p}_{1}-\frac{\theta_{2}}{2} \mathrm{n}_{1}+\frac{\theta_{4}}{2 \sqrt{12}} \mathrm{~m}_{1} \\
\beta_{+\mathrm{nc}}= \pm \frac{\mathrm{n}_{1}}{3 \mathrm{a}_{1}} \operatorname{Ln}\left[\frac{16 \mathrm{a}_{1}+\mathrm{b}_{0} \mathrm{t}}{\mathrm{t}}\right]+\frac{\theta_{1}}{2} \sqrt{\mathrm{a}_{1}^{2}+\frac{\mathrm{b}_{0} \mathrm{t}^{2}}{64}+\frac{\mathrm{a}_{1} \mathrm{t}}{4}}-\frac{\theta_{3}}{2} \mathrm{p}_{1}-\frac{\theta_{6}}{2 \sqrt{12}} \mathrm{~m}_{1} \\
\beta_{-\mathrm{nc}}= \pm \frac{\mathrm{p}_{1}}{3 \mathrm{a}_{1}} \operatorname{Ln}\left[\frac{16 \mathrm{a}_{1}+\mathrm{b}_{0} \mathrm{t}}{\mathrm{t}}\right]+\frac{\theta_{2}}{2} \sqrt{\mathrm{a}_{1}^{2}+\frac{\mathrm{b}_{0} \mathrm{t}^{2}}{64}+\frac{\mathrm{a}_{1} \mathrm{t}}{4}}+\frac{\theta_{3}}{2} \mathrm{n}_{1}-\frac{\theta_{5}}{2 \sqrt{12}} \mathrm{~m}_{1} \\
\phi= \pm \frac{4 \mathrm{~m}_{1}}{\sqrt{6} \mathrm{a}_{1}} \operatorname{Ln}\left[\frac{16 \mathrm{a}_{1}+\mathrm{b}_{0} \mathrm{t}}{\mathrm{t}}\right]-\frac{\theta_{4}}{2} \sqrt{\mathrm{a}_{1}^{2}+\frac{\mathrm{b}_{0} \mathrm{t}^{2}}{64}+\frac{\mathrm{a}_{1} \mathrm{t}}{4}}+\frac{\theta_{5}}{2} \mathrm{n}_{1}+\frac{\theta_{6}}{2} \mathrm{p}_{1}\end{array}$ \\
\hline
\end{tabular}

if we proceed as in the commutative case we get the solutions showed in the table IV.

Table IV Noncommutative solutions for, $\gamma=-1,1,0$, and constraints $q$, $a_{1}$ and $b_{0}$.

We see that the noncommutativity parameters $\theta_{1}$ and $\theta_{2}$ could be chosen, with same fine tunning, in order to lower the anisotropy at the present time, but in the long term the anisotripy will reappear. The evolution of the isotropy (anisotropy) can be appreciate in figure (2).

\section{NONCOMMUTATIVE QUANTUM SOLUTION}

As already mentioned, we are looking for the noncommuative deformation of the Bianchi I quantum cosmological model. In order to find the noncommutative generalization we need to solve the noncommutative Einstein equation, 

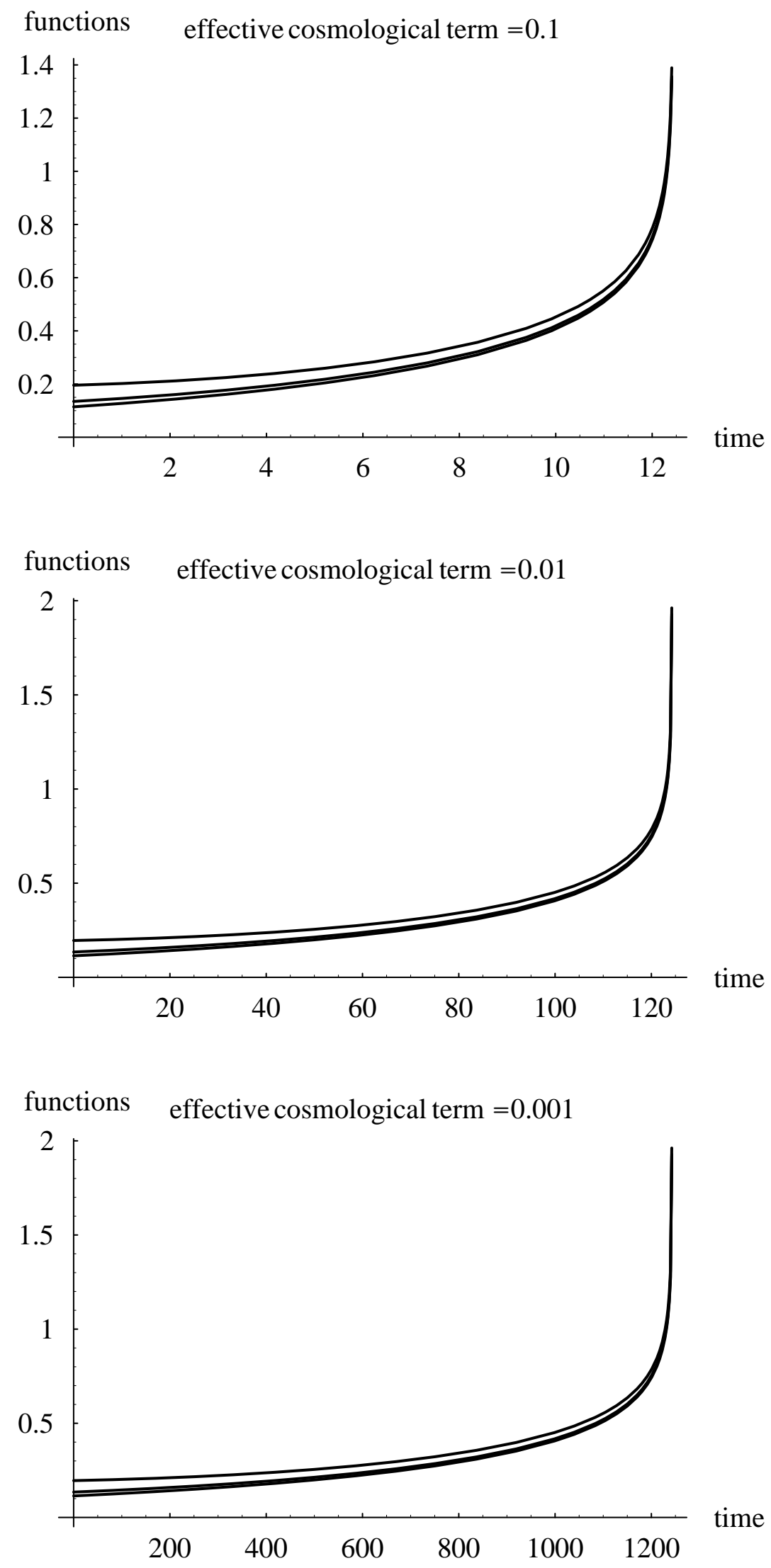

FIG. 2: Plots of $\beta_{ \pm \mathrm{nc}}$ and $\phi$ field, that appear in the second line in the table IV using the values in the parameters $\mathrm{n}_{1}=$ $1, \mathrm{p}_{1}=1, \mathrm{~m}_{1}=1$ and $\theta=0,0.05,0.2$, from bottom to top in the figure. The possible isotropization in the parameters $\beta_{ \pm n c}$ and the decay in the $\phi_{n c}$ field is saw in function of the curvature, but it appears again in this fields after critical cosmic time $t_{c}$ 
this is a formidable task, due to the highly non linear character of the theory [5], fortunately we can circumvent these difficulties by following Ref. [4]. Now we can proceed to the non commutative model, actually we will consider, that the minisuperspace variables $\mathrm{q}^{\mathrm{i}}=\left(\Omega, \beta_{+}, \beta_{-}, \phi\right)$ do not commute, so that the symplectic structure is modified as follows

$$
\left[\mathrm{q}^{\mathrm{i}}, \mathrm{q}^{\mathrm{j}}\right]=\mathrm{i} \theta^{\mathrm{ij}}, \quad\left[\mathrm{P}_{\mathrm{i}}, \mathrm{P}_{\mathrm{j}}\right]=0, \quad\left[\mathrm{q}^{\mathrm{i}}, \mathrm{P}_{\mathrm{j}}\right]=\mathrm{i} \delta_{\mathrm{j}}^{\mathrm{i}}
$$

in particular, we choose the following representation

$$
\begin{aligned}
{\left[\Omega, \beta_{+}\right] } & =\mathrm{i} \theta_{1}, \quad\left[\Omega, \beta_{-}\right]=\mathrm{i} \theta_{2}, \quad\left[\beta_{-}, \beta_{+}\right]=\mathrm{i} \theta_{3}, \\
{[\Omega, \phi] } & =\mathrm{i} \theta_{4}, \quad\left[\phi, \beta_{+}\right]=\mathrm{i} \theta_{5}, \quad\left[\phi, \beta_{-}\right]=\mathrm{i} \theta_{6}
\end{aligned}
$$

where the $\theta_{\mathrm{i}}$ parameters are a measure of the non commutativity between the minisuperspace variables. The commutation relation (58) or (59) are not the most general deformations that define a noncommutative field; but this relations give us the isotropization of the models that we are working.

It is well known, that this non-commutativity can be formulated in term of non-commutative minisuperspace functions with the Moyal star product $\star$ of functions. The commutation relations (58) can be implemented in terms of the commuting coordinates of the standard quantum mechanics ( Bopp shift) and it results in a modification of the potential term of the WDW equation [4, 10], and one possibility is, for example,

$$
\begin{aligned}
\Omega_{\mathrm{nc}} & \rightarrow \Omega+\frac{\theta_{1}}{2} \mathrm{P}_{+}+\frac{\theta_{2}}{2} \mathrm{P}_{-}+\frac{\theta_{4}}{2} \mathrm{P}_{\phi}, \\
\beta_{-\mathrm{nc}} & \rightarrow \beta_{-}-\frac{\theta_{2}}{2} \mathrm{P}_{\Omega}+\frac{\theta_{3}}{2} \mathrm{P}_{+}-\frac{\theta_{6}}{2} \mathrm{P}_{\phi}, \\
\beta_{+\mathrm{nc}} & \rightarrow \beta_{+}-\frac{\theta_{1}}{2} \mathrm{P}_{\Omega}-\frac{\theta_{3}}{2} \mathrm{P}_{-}-\frac{\theta_{5}}{2} \mathrm{P}_{\phi}, \\
\phi_{\mathrm{nc}} & \rightarrow \phi-\frac{\theta_{4}}{2} \mathrm{P}_{\Omega}+\frac{\theta_{5}}{2} \mathrm{P}_{+}+\frac{\theta_{6}}{2} \mathrm{P}_{-} .
\end{aligned}
$$

These transformation are not the most general in order to define noncommutative fields, we can see for example Carmona et al. [11], where the authors give the generalization of the noncommutative harmonic oscillator and have shown the most linear transformation between the coordinates and momenta. In Vakili et al. [12] a similar procedure is applied to Bianchi I models without matter.

However, these shifts modify the potential term as follows

$$
\begin{aligned}
& \mathrm{U}\left(\Omega, \beta_{ \pm}, \phi, \theta_{\mathrm{i}}\right)=-48 \Lambda \mathrm{e}^{6\left[\Omega+\frac{\theta_{1}}{2} \mathrm{P}_{+}+\frac{\theta_{2}}{2} \mathrm{P}_{-}+\frac{\theta_{4}}{2} \mathrm{P}_{\phi}\right]}+384 \pi \mathrm{GM}_{\gamma} \mathrm{e}^{-3(\gamma-1)\left[\Omega+\frac{\theta_{1}}{2} \mathrm{P}_{+}+\frac{\theta_{2}}{2} \mathrm{P}_{-}+\frac{\theta_{4}}{2} \mathrm{P}_{\phi}\right]} \\
& +12 \mathrm{e}^{6\left[\Omega+\frac{\theta_{1}}{2} \mathrm{P}_{+}+\frac{\theta_{2}}{2} \mathrm{P}_{-}+\frac{\theta_{4}}{2} \mathrm{P}_{\phi}\right]} \mathrm{V}\left(\phi-\frac{\theta_{4}}{2} \mathrm{P}_{\Omega}+\frac{\theta_{5}}{2} \mathrm{P}_{+}+\frac{\theta_{6}}{2} \mathrm{P}_{-}\right)
\end{aligned}
$$

Using the generalized Baker-Campbell-Hausdorff formula [22],

$$
\mathrm{e}^{\eta(\hat{\mathrm{A}}+\hat{\mathrm{B}})}=\mathrm{e}^{-\eta^{2}[\hat{\mathrm{A}}, \hat{\mathrm{B}}]} \mathrm{e}^{\eta \hat{\mathrm{A}}} \mathrm{e}^{\eta \hat{\mathrm{B}}}
$$

and the relation between the variables (59), we find the noncommutative WDW (NCWDW) equation

$$
\begin{aligned}
& {\left[\frac{\partial^{2}}{\partial \Omega^{2}}-\frac{\partial^{2}}{\partial \beta_{+}^{2}}-\frac{\partial^{2}}{\partial \beta_{-}^{2}}-12 \frac{\partial^{2}}{\partial \phi^{2}}+Q \frac{\partial}{\partial \Omega}-48 \Lambda e^{6\left[\Omega-\frac{i \theta_{1}}{2} \partial_{+}-\frac{i \theta_{2}}{2} \partial_{-}-\frac{i \theta_{4}}{2} \partial_{\phi}\right]}\right.} \\
& +384 \pi \mathrm{GM}_{\gamma} \mathrm{e}^{-3(\gamma-1)\left[\Omega-\frac{\mathrm{i} \theta_{1}}{2} \partial_{+}-\frac{\mathrm{i} \theta_{2}}{2} \partial_{-}-\frac{\mathrm{i} \theta_{4}}{2} \partial_{\phi}\right]}+12 \mathrm{e}^{6\left[\Omega-\frac{\mathrm{i} \theta_{1}}{2} \partial_{+}-\frac{\mathrm{i} \theta_{2}}{2} \partial_{-}-\frac{\mathrm{i} \theta_{4}}{2} \partial_{\phi}\right]_{X}} \\
& \left.\mathrm{~V}\left(\phi+\frac{\mathrm{i} \theta_{4}}{2} \partial_{\Omega}-\frac{\mathrm{i} \theta_{5}}{2} \partial_{+}-\frac{\mathrm{i} \theta_{6}}{2} \partial_{-}\right) \cdot\right] \Psi=0
\end{aligned}
$$


To solve the equation we are going to focus our efforts on the same cases that were considered in the commutative model.

Lets start with case $1, \Lambda_{\text {eff }}=0, \gamma \neq 1$, and factor ordering $\mathrm{Q} \neq 0$. We propose an ansatz of the form

$$
\Psi\left(\Omega, \beta_{ \pm}, \phi\right) \equiv \mathrm{e}^{ \pm \mathrm{i} \eta \phi} \mathrm{e}^{ \pm \mathrm{i} \nu \beta_{+}} \mathrm{e}^{ \pm \mathrm{i} \mu \beta_{-}} \mathcal{A}(\Omega)
$$

and taking in account that $\mathrm{e}^{\mathrm{i} \theta \frac{\partial}{\partial \mathrm{x}}} \mathrm{e}^{\eta \mathrm{x}} \equiv \mathrm{e}^{\mathrm{i} \eta \theta} \mathrm{e}^{\eta \mathrm{x}}$, then equation (63) is written as

$$
\frac{\mathrm{d}^{2} \mathcal{A}}{\mathrm{d} \Omega^{2}}+\mathrm{Q} \frac{\mathrm{d} \mathcal{A}}{\mathrm{d} \Omega}+\left(\alpha^{2}+384 \pi \mathrm{GM}_{\gamma} \mathrm{e}^{-3(\gamma-1) \Omega} \mathrm{e}^{\mp \frac{3}{2}(\gamma-1)\left(\theta_{4} \eta+\theta_{1} \nu+\theta_{2} \mu\right.}\right) \mathcal{A}=0
$$

where the separation constants have the following relation between them, $\mu^{2}=\alpha^{2}-\nu^{2}-12 \eta^{2}$. This equation can be written as an ordinary Bessel equation by making the transformation $\mathrm{z}=\mathrm{z}_{0} \mathrm{e}^{-3(\gamma-1) \Omega}$, with $\mathrm{z}_{0}=$ $384 \pi \mathrm{GM}_{\gamma} \mathrm{e}^{\mp \frac{3}{2}(\gamma-1)\left(\theta_{4} \eta+\theta_{1} \nu+\theta_{2} \mu\right)}$,

$$
\mathrm{z}^{2} \frac{\mathrm{d}^{2} \mathcal{A}}{\mathrm{dz}^{2}}+\left(1+\frac{\mathrm{Q}}{9(\gamma-1)^{2}}\right) \mathrm{z} \frac{\mathrm{d} \mathcal{A}}{\mathrm{dz}}+\frac{1}{9(\gamma-1)^{2}}\left(\mathrm{z}+\alpha^{2}\right) \mathcal{A}=0
$$

whose solution is

$$
\mathcal{A}_{\rho}=\mathrm{z}^{\mathrm{q}_{0}} \mathrm{~J}_{ \pm \mathrm{i} \rho}\left(\frac{2}{3(\gamma-1)} \mathrm{z}^{\frac{1}{2}}\right), \quad \mathrm{q}_{0}=-\frac{\mathrm{Q}}{18(\gamma-1)^{2}}
$$

with order $\rho=\frac{1}{3(\gamma-1)} \sqrt{4 \alpha^{2}-\left(\frac{\mathrm{Q}}{3(\gamma-1)}\right)^{2}}$. As expected, in the limit $\theta_{i} \rightarrow 0$ we get the commutative solutions, so the transition from noncommutative minisuperspace is well defined.

For case $2, \Lambda_{\text {eff }} \neq 0, \gamma=1$, and factor ordering $\mathrm{Q} \neq 0$, the NCWDW equation has the "noncommutative potential" $\mathrm{U}\left(\Omega, \Lambda_{\text {eff }}, \theta\right)=\left(\Lambda_{\text {eff }} \mathrm{e}^{6\left[\Omega-\frac{\mathrm{i} \theta_{1}}{2} \partial_{+}-\frac{\mathrm{i} \theta_{2}}{2} \partial_{-}-\frac{\mathrm{i} \theta_{4}}{2} \partial_{\phi}\right]}+\mathrm{c}_{1}\right)$. After separating with $\Psi \equiv \mathrm{e}^{ \pm \mathrm{i} \eta \phi} \mathrm{e}^{ \pm \mathrm{i} \nu \beta_{+}} \mathrm{e}^{ \pm \mathrm{i} \mu \beta_{-}} \mathcal{A}(\Omega)$, we get

$$
\frac{\mathrm{d}^{2} \mathcal{A}}{\mathrm{d} \Omega^{2}}+\mathrm{Q} \frac{\mathrm{d} \mathcal{A}}{\mathrm{d} \Omega}+\left(\Lambda_{\text {eff }} \mathrm{e}^{6 \Omega} \mathrm{e}^{\mathrm{f}\left(\theta_{\mathrm{i}}\right)}+\mathrm{c}_{1}\right) \mathcal{A}=0
$$

where the constant $\mathrm{c}_{1}$ is the same, as in the commutative case, and $\mathrm{f}\left(\theta_{\mathrm{i}}\right)=\mp 3\left(\theta_{4} \eta+\theta_{1} \nu+\theta_{2} \mu\right)$. The solution for equation (68) is written with the generic Bessel function, making the transformation $\xi=\Lambda_{\text {eff }} \mathrm{e}^{\mp 3\left(\theta_{4} \eta+\theta_{1} \nu+\theta_{2} \mu\right)} \mathrm{e}^{6 \Omega}$,

$$
\mathcal{A}(\xi)=\xi^{-\frac{\mathrm{Q}}{12}} \mathrm{Z}_{\rho}\left( \pm \frac{1}{3} \xi^{\frac{1}{2}}\right)
$$

with order $\rho=\frac{1}{6} \sqrt{\mathrm{Q}^{2}-4 \mathrm{c}_{1}}$.

As in the commutative case, for the simplest factor ordering, $Q=0$, simplified solutions are obtained, also the same restrictions on $\Lambda_{\text {eff }}$ appear.

For case $3, \Lambda_{\text {eff }} \neq 0, \gamma=-1$, and factor ordering $\mathrm{Q} \neq 0$, the NCWDW equation has the "noncommutative potential" $\mathrm{U}\left(\Omega, \Lambda_{\text {eff }}, \theta\right)=\mathrm{be}^{6\left[\Omega-\frac{\mathrm{i} \theta_{1}}{2} \partial_{+}-\frac{\mathrm{i} \theta_{2}}{2} \partial_{-}-\frac{\mathrm{i} \theta_{4}}{2} \partial_{\phi}\right]}$, with $\mathrm{b}=384 \pi \mathrm{GM}_{-1}+\Lambda_{\text {eff }}$. After doing a separation of variables, using $\Psi=\mathrm{e}^{ \pm \mathrm{i} \eta \phi} \mathrm{e}^{ \pm \mathrm{i} \nu \beta_{+}} \mathrm{e}^{ \pm \mathrm{i} \mu \beta_{-}} \mathcal{A}$, and proceeding as before, we make the transformation $\xi=\mathrm{be}^{\mp 3\left(\theta_{4} \eta+\theta_{1} \nu+\theta_{2} \mu\right)} \mathrm{e}^{6 \Omega}$, we arrive to the equation

$$
\xi^{2} \frac{\mathrm{d}^{2} \mathcal{A}}{\mathrm{d} \xi^{2}}+\left(1+\frac{\mathrm{Q}}{6}\right) \xi \frac{\mathrm{d} \mathcal{A}}{\mathrm{d} \xi}+\frac{1}{36}\left(\xi+\alpha^{2}\right) \mathcal{A}=0
$$




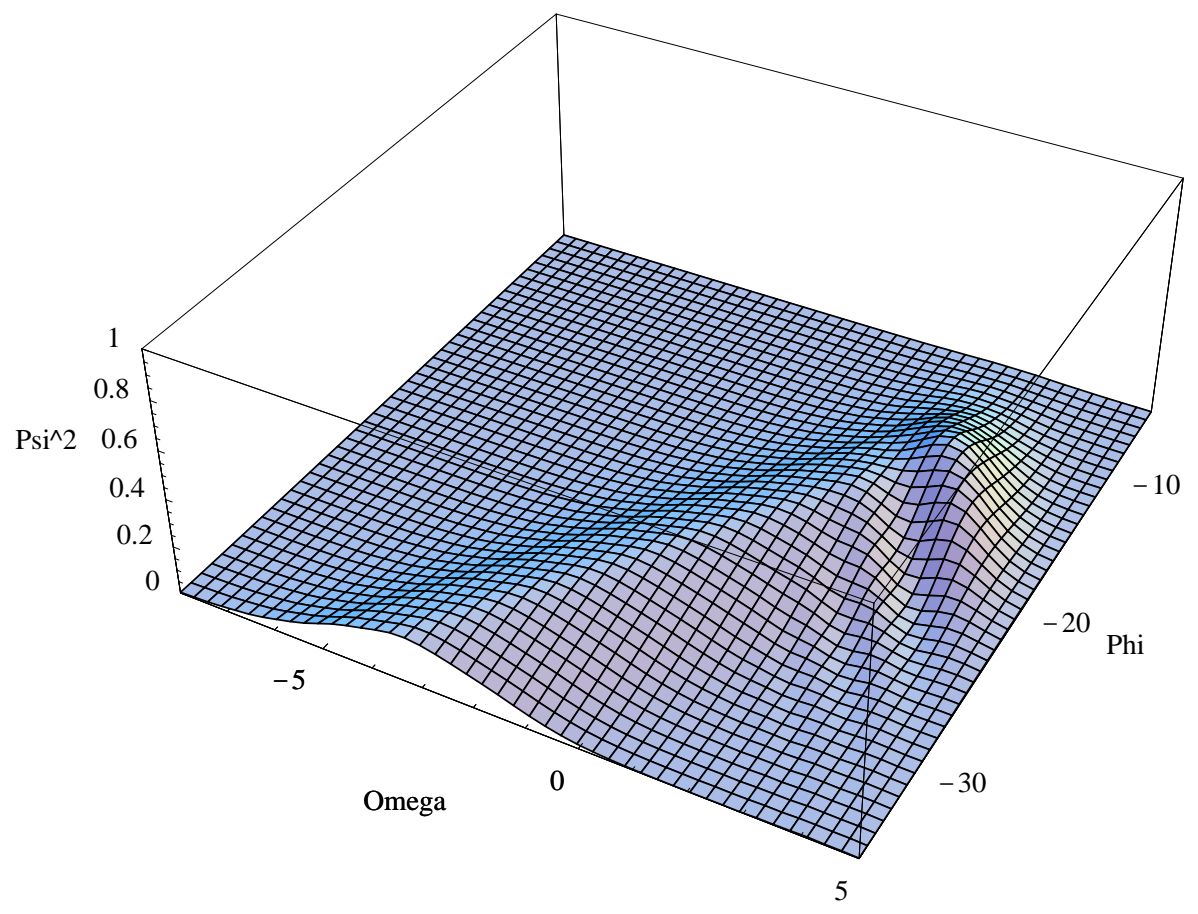

FIG. 3: 3D plot for $(\Omega, \phi)$, corresponding to the Gaussian wavepacket equation (72) in the noncommuting case using $\theta=4$ and $\mu=\nu=0$.

the solution is written with generic Bessel functions

$$
\mathcal{A}(\xi)=\xi^{-\frac{Q}{12}} \mathrm{Z}_{ \pm \mathrm{i} \rho}\left( \pm \frac{1}{3} \xi^{\frac{1}{2}}\right)
$$

with order $\rho=\frac{1}{6} \sqrt{4 \alpha^{2}-\mathrm{Q}^{2}}$.

The Gaussian weighted wavepacket for the particular factor ordering $\mathrm{Q}=0$ is

$$
\Psi(\Omega, \phi)=\mathcal{N} \int_{-\infty}^{\infty} \mathrm{e}^{-\mathrm{a}(\rho-\mathrm{b})^{2}} \mathrm{e}^{-\frac{\sqrt{3}}{2} \rho \phi} \mathrm{J}_{\mathrm{i} \rho}\left(\frac{1}{3} \sqrt{\mathrm{b}} \mathrm{e}^{3 \Omega} \mathrm{e}^{\frac{3 \sqrt{3}}{4} \rho \theta_{4}}\right) \mathrm{d} \rho,
$$

and the plot for $|\Psi|^{2}$ can be see in figures (34) taking two values for the noncomutative parameter $\theta=4,0.5$ respectively. Here again we have dropped the $\beta_{ \pm}$variables. As in the commutative case, we can to change the variable $\phi \rightarrow \beta_{ \pm}$, and we obtain similar behaviour.

The new structure in the probability density can be see in the figures (5) and (6), in the first along the line of the main structure in figures (1) and (3) when $\theta=4$, second in the line $\Omega=1.7$ presenting a peak in the variable $\phi$.

Finally, for case $\mathrm{V}(\phi)=\mathrm{e}^{-\beta \phi}$, stiff fluid $\gamma=1$ and null cosmological term, the NCWDW becomes

$$
\begin{aligned}
& {\left[\frac{\partial^{2}}{\partial \Omega^{2}}-\frac{\partial^{2}}{\partial \beta_{+}^{2}}-\frac{\partial^{2}}{\partial \beta_{-}^{2}}-12 \frac{\partial^{2}}{\partial \phi^{2}}+\mathrm{Q} \frac{\partial}{\partial \Omega}+384 \pi \mathrm{GM}_{1}+12 \mathrm{~V}_{0} \mathrm{e}^{6\left[\Omega-\frac{\mathrm{i} \theta_{1}}{2} \partial_{+}-\frac{\mathrm{i} \theta_{2}}{2} \partial_{-}-\frac{\mathrm{i} \theta_{4}}{2} \partial_{\phi}\right]} \times\right.} \\
& \left.\mathrm{e}^{-\beta\left[\phi+\frac{\mathrm{i} \theta_{4}}{2} \partial_{\Omega}-\frac{\mathrm{i} \theta_{5}}{2} \partial_{+}-\frac{\mathrm{i} \theta_{6}}{2} \partial_{-}\right]}\right] \Psi=0 \text {. }
\end{aligned}
$$

Choosing the wave function $\Psi=\mathrm{e}^{ \pm \mathrm{i} \nu \beta_{+}} \mathrm{e}^{\mathrm{i} \mu \beta_{-}} \mathcal{F}(\Omega, \phi)$, we have

$$
\frac{\partial^{2} \mathcal{F}}{\partial \Omega^{2}}-12 \frac{\partial^{2} \mathcal{F}}{\partial \phi^{2}}+\mathrm{Q} \frac{\partial \mathcal{F}}{\partial \Omega}+\left[\nu^{2}+\mu^{2}+384 \pi \mathrm{GM}_{1}+12 \mathrm{~V}_{0} \mathrm{e}^{\mathrm{h}\left(\theta_{\mathrm{i}}\right)} \mathrm{e}^{6 \Omega-\beta \phi} \mathrm{e}^{-\frac{\mathrm{i} \theta_{4}}{2}\left[\beta \partial_{\Omega}+6 \partial_{\phi}\right]}\right] \mathcal{F}=0
$$




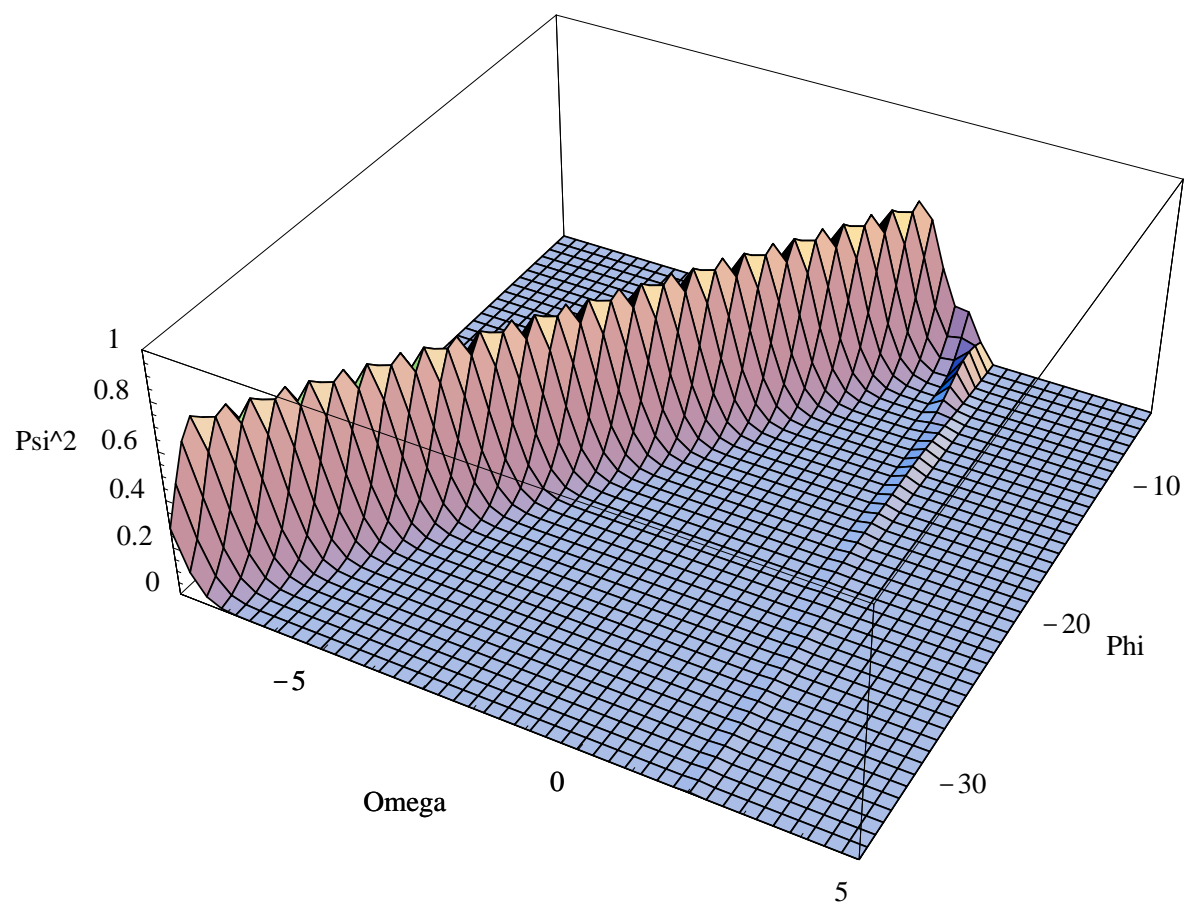

FIG. 4: $3 \mathrm{D}$ plot for $(\Omega, \phi)$, corresponding to the Gaussian wavepacket equation (72) in the noncommuting case using $\theta=0.5$ and $\mu=\nu=0$.

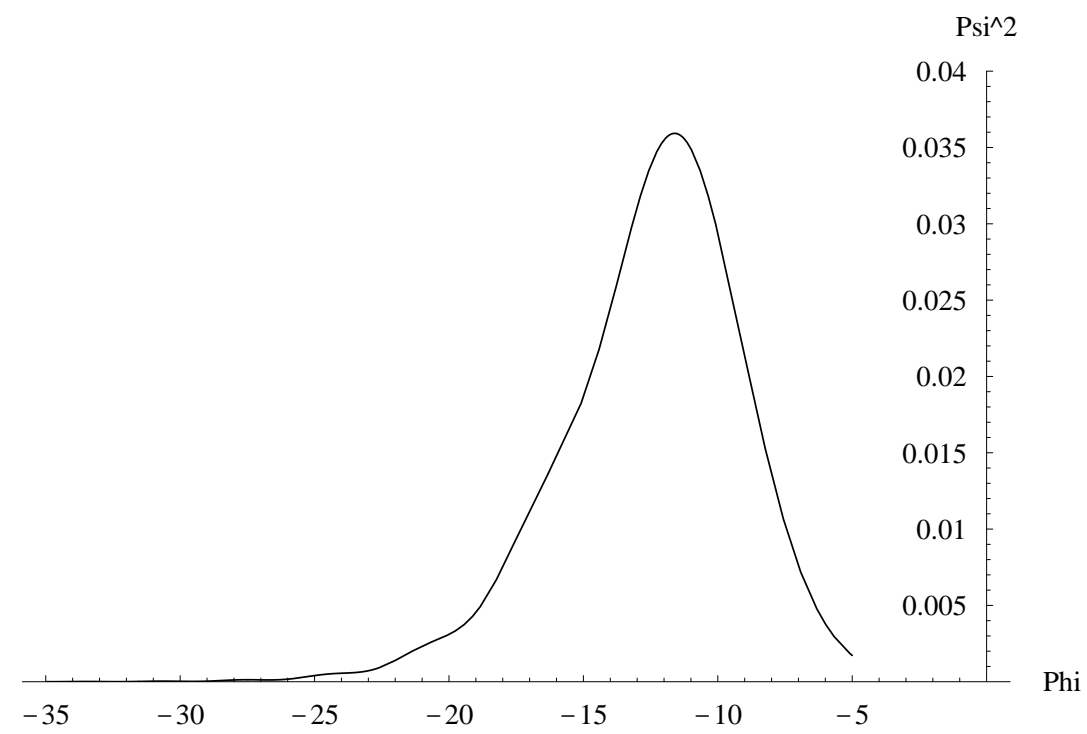

FIG. 5: In this plot we present the variation of $|\Psi|^{2}$ with respect to $\phi$ when $\theta=0.5$ along the line of $\Omega=1.7$ in figure (4).

where $\mathrm{h}\left(\theta_{\mathrm{i}}\right)=\left(3 \theta_{1}-\frac{\beta}{2} \theta_{5}\right) \nu+\left(3 \theta_{2}-\frac{\beta}{2} \theta_{6}\right) \mu$.

It is easier to work in the new variables $(\mathrm{x}, \mathrm{y})$ given by (38), in place of $(\Omega, \phi)$. Thus we have the corresponding differential equation

$$
12\left(3-\beta^{2}\right) \frac{\mathrm{d}^{2} \mathcal{F}}{\mathrm{dx}^{2}}-6 \mathrm{Q} \frac{\mathrm{d} \mathcal{F}}{\mathrm{dx}}+\left[12 \mathrm{~V}_{0} \mathrm{e}^{\mathrm{h}\left(\theta_{\mathrm{i}}\right)} \mathrm{e}^{-\mathrm{x}}+\mathrm{c}\right] \mathcal{F}+\frac{4\left(\beta^{2}-3\right)}{\beta^{2}} \frac{\mathrm{d}^{2} \mathcal{F}}{\mathrm{dy}^{2}}-2 \mathrm{Q} \frac{\mathrm{d} \mathcal{F}}{\mathrm{dy}}=0
$$




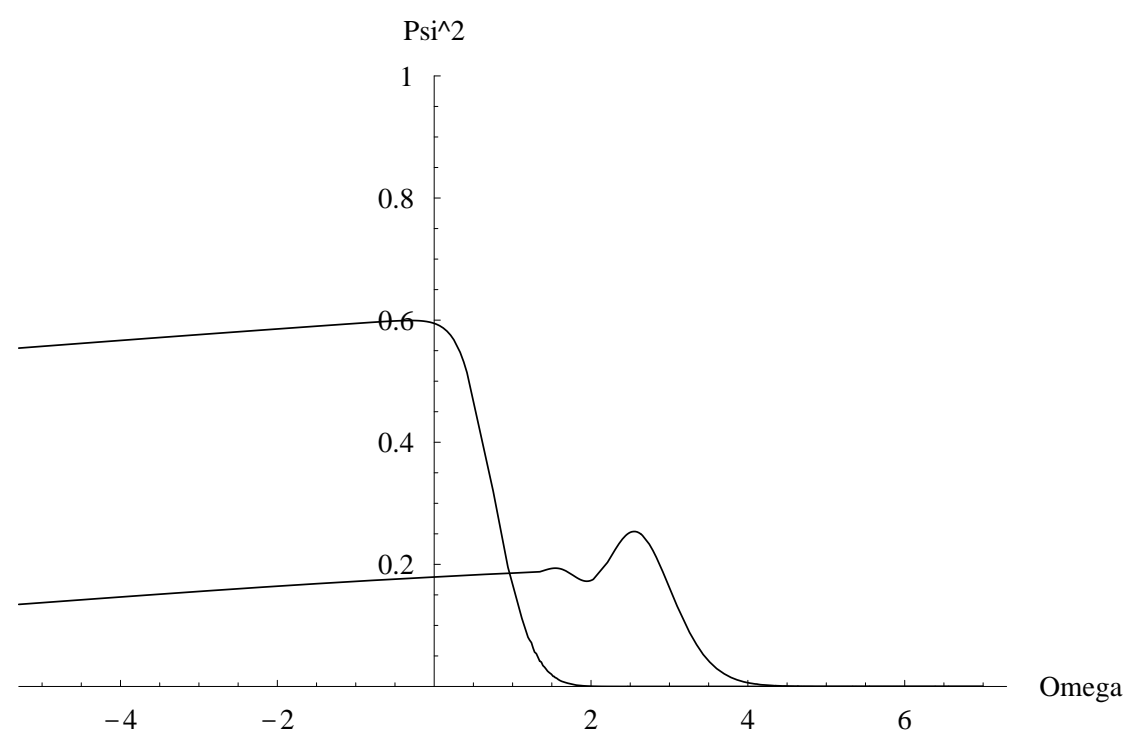

FIG. 6: In these plots we present the variation of $|\Psi|^{2}$ with respect to $\Omega$ when $\theta=0$ (commutative universe, big plot) and $\theta=4$ (no commutative universe, small plot), along the line of the main structure in figures 1 and 3 .

For solving the equation (75) we choose the ansatz $\mathcal{F}(\mathrm{x}, \mathrm{y})=\mathrm{e}^{(\mathrm{s}-\ell) \mathrm{y}} \mathrm{X}(\mathrm{x})$, where $\ell=\frac{Q \beta^{2}}{4\left(\beta^{2}-3\right)}$, obtaining the following equation for the function $\mathrm{X}$,

$$
\frac{\mathrm{d}^{2} \mathrm{X}}{\mathrm{dx}^{2}}-\frac{\mathrm{Q}}{2\left(3-\beta^{2}\right)} \frac{\mathrm{dX}}{\mathrm{dx}}++\frac{1}{12\left(3-\beta^{2}\right)}\left[12 \mathrm{~V}_{0} \mathrm{e}^{\mathrm{f}\left(\theta_{\mathrm{i}}\right)} \mathrm{e}^{-\mathrm{x}}+\mathrm{n}^{2}\right] \mathrm{X}=0
$$

where $\mathrm{n}^{2}=\mathrm{c}-\mathrm{m}^{2}, \mathrm{~m}^{2}=2 \mathrm{Q}(\mathrm{s}-\ell)+\frac{4}{\beta^{2}}\left(3-\beta^{2}\right)(\mathrm{s}-\ell)^{2}$ and $\mathrm{f}\left(\theta_{\mathrm{i}}\right)=\mathrm{h}\left(\theta_{\mathrm{i}}\right)-\mathrm{i} \frac{\theta_{4}}{\beta}\left(3-\beta^{2}\right)(\mathrm{s}-\ell)$ and $\mathrm{s}$ is the constant that appears in the commutative case.

This equation is similar to (39), then the solution is similar, and only suffers a shift in the argument of the Bessel function

$$
\mathrm{X}(\mathrm{x})=\operatorname{Exp}\left[-\frac{\mathrm{Q}}{4\left(3-\beta^{2}\right)} \mathrm{x}\right] \mathrm{J}_{ \pm \mathrm{r}}\left(\sqrt{\frac{\mathrm{V}_{0}}{3-\beta^{2}}} \mathrm{e}^{\mathrm{f}\left(\theta_{\mathrm{i}}\right)} \mathrm{e}^{-\mathrm{x} / 2}\right)
$$

For the remaining case, $\beta^{2}=3$ the solution to the differential equation is

$$
\mathcal{F}=F_{1} e^{-\frac{Q \Omega}{2}}\left[e^{\frac{6 d x+a e^{6 x}}{6 \kappa}} e^{-\frac{\kappa y}{4}}\right] .
$$

here $\kappa$ is a non null separation constant, $F_{1}$ is an arbitrary integration constant, $a=c-Q^{2} / 4, d=12 V_{0} e^{m\left(\theta_{i}\right)}=$ $b e^{m\left(\theta_{i}\right)}$, with

$$
m\left(\theta_{i}\right)=\left(3 \theta_{1} \mp \frac{\sqrt{3}}{2} \theta_{5}\right) \nu+\left(3 \theta_{2} \mp \frac{\sqrt{3}}{2} \theta_{6}\right) \mu \mp \frac{\sqrt{3}}{4} \theta_{4} \kappa .
$$

and the variables $x$ and $y$ are defined as in the commutative case (equation (44)).

\section{FINAL REMARKS}

In this work by using the equivalence between General Relativity and Hamiltonian formalism, noncommutative scenarios are constructed. This was achieved by deforming the minisuperspace for the Bianchi type I cosmological 
model coupled to barotropic perfect fluid, field $\phi$ and cosmological term. in the gauge $\mathrm{N}=1$, as we can see the solution $\Omega_{\mathrm{nc}}$ is the commutative solution plus a function on $\theta_{i}$, independent of time. We have also analyzed the $\beta_{ \pm \mathrm{nc}}$ noncommutative solutions, in some ranges on the parameter $\theta_{i}$ and effective cosmological constant, occurs a dynamical isotropization, i.e., $\beta_{ \pm n c}$ are bounded in the present time, noting that different evolution with respect to the commutative $\beta_{ \pm}$, also the field $\phi$ decays dynamically to a constant, but after a critical time $t_{c}$ the evolution is the same in both scenarios. In the quantum stadium, in all but one of the cases considered the influence of the noncommutativity is encoded in a change of the argument of Bessel functions and the change is not qualitatively very remarkable ( see figures (1136). The singular case is the one with $\beta^{2}=3$ and here the influence of the noncommutativity is in the argument of an exponential in the form $6 d x+a e^{6 x}$. Only the constant $\mathrm{d}$ depends on the noncommutivity parameters and therefore we have that for $x$ large and negative they will dominate and for large positive $x$ the noncommutative effects disappear. From the definition of $x$ we see that for finite $\phi$ the noncommutative regime corresponds to $\Omega \rightarrow-\infty$ and at late times commutativity is recovered. It would be of interest to improve the work with the two approaches cited in the references [14, 15], and obtain the possibles differences in the dynamics of our universe, using anisotropic cosmological models; this will be reported in future work.

\section{Acknowledgments}

This work was supported in part by CONACyT grant 47641, DINPO, CONCYTEG and Promep grant UGTO-CA3. Many calculations where done by Symbolic Program REDUCE 3.8. We also like to thank M. Sabido for usefull comments. This work is part of the collaboration within the Instituto Avanzado de Cosmología.

[1] M.P. Ryan, Hamiltonian cosmology, (Springer, Berlin, 1972).

[2] M.P. Ryan and L.C. Shepley, Homogeneous Relativistic Cosmologies, Princeton University Press, Princeton, New Jersey (1975).

[3] M. MacCallum, in: General Relativity: An Einstein Centenary Survey, edited by S. Hawking and W. Israel (Cambridge University Press, Cambridge, England, 1979).

[4] H. Garcia-Compean, O. Obregón and C. Ramírez, Phys. Rev. Lett. 88, 161301 (2002).

[5] H. García-Compeán, O. Obregón, C. Ramírez and M. Sabido, Phys. Rev. D 68 (2003) 044015; H. García-Compeán, O. Obregón, C. Ramírez and M. Sabido, Phys. Rev. D 68 (2003) 045010; M. Bañados, O. Chandia, N. Grandi, F.A. Schaposnik and G.A. Silva, Phys. Rev. D 64 (2001) 084012; H. Nishino and S. Rajpoot, Phys. Lett. B 532 (2002) 334 ; V.P. Nair, "Gravitational Fields on a Noncommutative Space", hep-th/0112114; S. Cacciatori, D. Klemm, L. Martucci and D. Zanon, Phys. Lett. B 536 (2002) 101; S. Cacciatori, A.H. Chamseddine, D. Klemm, L. Martucci, W.A. Sabra and D. Zanon, "Noncommutative Gravity in Two Dimensions", hep-th/0203038; Y. Abe and V.P. Nair, "Noncommutative Gravity: Fuzzy Sphere and Others", hep-th/0212270 M.A. Cardella and Daniela Zanon, "Noncommutative Deformation of Fourdimensional Einstein Gravity", hep-th/0212071; A.H. Chamseddine, "Invariant Actions for Noncommutative Gravity", hep-th/0202137; J.W. Moffat, Phys. Lett. B 491 (2000) 345; Phys. Lett. B 493 (2000) 142; V. O. Rivelles, Phys. Lett. B 558 (2003) 19; E. Harikumar and V. O. Rivelles, arXiv:hep-th/0607115. Class. Quantum Grav. 26065003 (2009) arXiv:0901.3829.

[6] M. Chaichian, M. M. Sheikh-Jabbari, and A. Tureanu., Phys. Rev. Lett. 86, 2716 (2001).

[7] J. Gamboa, M. Loewe and J. C. Rojas, Phys. Rev D 64, 067901 (2001).

[8] G.D. Barbosa, Phys. Rev. D 71, 063511 (2005), arXiv:hep-th/0408071.

[9] G.D. Barbosa and N. Pinto-Neto, Phys. Rev. D 70, 103512 (2004), arXiv:hep-th/0407111].

[10] L.O. Pimentel and C. Mora, Gen. Rel. Grav. 37, 817 (2005), arXiv:gr-qc/0408100.

[11] J.M. Carmona, J.L. Cortes, J. Gamboa, and F. Mendez, Quantum theory of noncommutative fields, JHEP 0303, 058 (2003). arXiv:hep-th/0301248.

[12] B. Vakili, N. Khosravi, H.R. Sepangi, Bianchi spacetimes in noncommutative phase-space, Class. Quantum Grav. 24, 931 (2007). arXiv:gr-qc/0701075.

[13] C. Ortiz, E. Mena, M. Sabido and J. Socorro, Int. J. Theor. Phys. 47 (5), 1240 (2008). (Non) commutative isotropization in Bianchi I with barotropic perfect fluid and $\Lambda$ cosmological. gr-qc/0703101. 
[14] B. Vakili, H.R. Sepangi, Phys. Lett. B 651, 79 (2007) arXiv:0706.0273; B. Vakili, Cosmology with minimal length uncertainty relations, arXiv:0811.3481; B. Vakili, Phys. Rev. D 77, 044023 (2008), arXiv:0801.2438; B. Vakili, N. Khosravi, H.R. Sepangi, Int. J. Mod. Phys. D 18, 159 (2008) arXiv:0804.4326; H.R. Sepangi, B. Shakerin and B. Vakili, Class. Quantum Grav. 26, 065003 (2009).

[15] N.Khosravi, H.R. Sepangi, JCAP 0804, 011 (2008) arXiv:0803.1714; N.Khosravi, H.R. Sepangi, Phys. Lett. A 372, 3351 (2008) arXiv:0802.0767.

[16] J. Kowalski-Glikman and S. Nowak, Phys. Lett. B 539, 126 (2002); J. Kowalski-Glikman, Phys. Lett. A 299, 454 (2002).

[17] Enrique Pazos, tesis licenciatura: Aplicación del formalismo lagrangiano ADM a un modelo cosmológico, Univ. de San Carlos de Guatemala, (2000).

[18] J. Hartle and S.W. Hawking, Phys. Rev. D 28, 2960 (1983).

[19] R.J. Szabo, Phys. Rep. 378, 207 (2003), arXiv:hep-th/0109162.

[20] C. Kiefer, Phys. Rev. D 38, 1761 (1988); Nuc. Phys. B 341, 273 (1990).

[21] I.S. Gradshteyn and I.M. Ryshik, Table of Integrals, Series, and Product (Academic Press, 1980).

[22] R.M. Wilcox, J. Math. Phys. 8, 962 (1967). 